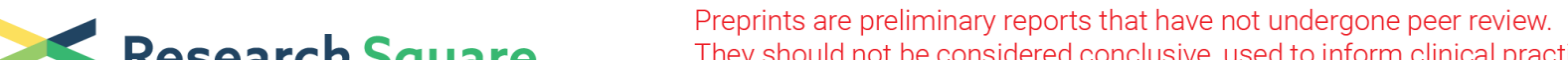 Research Square They should not be considered conclusive, used to inform clinical practice, or referenced by the media as validated information.
}

\section{Experimental Pulmonary Tuberculosis Without Brain Infection in Male BALB/c Mice Induces Neuroinflammation and Behavioural Abnormalities}

Jacqueline Viridiana Lara Espinosa

Instituto Nacional de Ciencias Medicas y Nutricion Salvador Zubiran https://orcid.org/0000-00016635-2939

Brenda Marquina-Castillo ( $\nabla$ brenda.marquinac@incmnsz.mx )

Ricardo A. Santana-Martínez

Universidad Nacional Autonoma de Mexico

Mario A. Zetter

Instituto Nacional de Ciencias Medicas y Nutricion Salvador Zubiran

Perla D. Maldonado

Instituto Nacional de Neurologia y Neurocirugia Manuel Velasco Suarez

Enrique Becerril-Villanueva

Instituto Nacional de Psiquiatria Ramon de la Fuente Muniz

Gilberto Pérez-Sánchez

Instituto Nacional de Psiquiatria Ramon de la Fuente Muniz

Lenin Pavón

Instituto Nacional de Psiquiatria Ramon de la Fuente Muniz

Dulce Mata-Espinosa

Instituto Nacional de Ciencias Medicas y Nutricion Salvador Zubiran

Jorge Barrios-Payán

Instituto Nacional de Ciencias Medicas y Nutricion Salvador Zubiran

Manuel Othoniel López-Torres

Instituto Nacional de Ciencias Medicas y Nutricion Salvador Zubiran

Rogelio Hernández Pando

Instituto Nacional de Ciencias Medicas y Nutricion Salvador Zubiran

Research

Keywords: Mycobacterium tuberculosis, neuroinflammation, behaviour abnormalities

Posted Date: May 4th, 2020

DOl: https://doi.org/10.21203/rs.3.rs-24798/v1 
License: (c) (i) This work is licensed under a Creative Commons Attribution 4.0 International License. Read Full License 


\section{Abstract}

Background During the development of chronic peripheral inflammation, there is a complex activity of specific brain areas that produce neuronal activation and changes in the synthesis, production and activity of neurotransmitters and cytokines. These changes produced afferent responses that contribute to the regulation of inflammatory and immune responses but may also affect the neuropsicoaffective functions, such as learning ability, memory and mood. Pulmonary tuberculosis (TB) is an infectious disease that induces chronic inflammation, so it could produce these neural and immune changes that may occur in the absence of brain infection. In the present study, we determined the immune response in the Central Nervous System (CNS), in a murine model of pulmonary TB and its relationship with the neurotransmitters levels and behavioural abnormalities without brain infection.

Methods Using a murine model of pulmonary TB, we studied the synthesis of cytokines by RT-PCR and $\mathrm{IHC}$, the concentrations of the neurotransmitters by HPLC along with neuronal changes and the histopathology alterations within specific brain areas. Different behavioural tests were performed, which include the study of sickness behaviour, anxiety-like behaviour, neurological severity score, short and long-term memory and depression-like behaviour. We evaluated the activation of JNK and p38 and BNDF levels by Western blot. Data were analysed by Two-Way ANOVA, followed by Sidak tests.

Results Pulmonary infected male animals showed a brain increased expression of the pro-inflammatory (TNFa, IFNg, IL-12) and anti-inflammatory (IL10, IL4 and TGFb) cytokines, as well as the enzymes indolamine 2-3 dioxygenase (IDO) and induced nitric oxide synthase (iNOS) in the hypothalamus, hippocampus and cerebellum; in coexistence with substantial cha nges in the production of the neurotransmitters norepinephrine, epinephrine, dopamine and serotonin. Neurodegenerative changes occurred in the early stage of the disease, and it led to neuronal death during advanced TB, with activation of p38, JNK and decrease of the BDNF levels. All these changes occurred with sickness behaviour, depressive-like and anxiety-like behaviour, neurological damage and impairment of both short and long-term memory.

Conclusions Pulmonary TB produced a neuroinflammatory state and neuronal damage, which evoked several behaviour abnormalities that resembled depression/anxiety states and aroused the interest to control neuroinflammation in TB patients with neuropsychiatric abnormalities.

\section{Background}

Tuberculosis (TB), an airborne infectious disease caused by bacteria of the Mycobacterium tuberculosis (Mtb) complex [1] is the principal killer among the infectious diseases (WHO report). The immune response to Mtb influences the TB clinical course and evokes a complex neuroendocrine response [2]. Indeed, TB patients exhibit numerous endocrine and immune abnormalities that contribute to the physiopathology of the disease [3]. These endocrine responses include abnormal production of several hormones, that is partly mediated by the cytokines released during the immune response to Mtb (pro- 
inflammatory mediators), and also contribute to modify the Central Nervous System (CNS) response by activating the two major stress systems, the hypothalamic-pituitary-adrenal (HPA) axis [4], the sympathetic nervous system (SNS) [2, 5] and the vasopressinergic system [6].

Previous studies have shown that chronic inflammatory diseases such as obesity, inflammatory bowel disease, chronic liver disease [7], and also mycobacterial infection, such as the systemic administration of the Bacillus Calmette-Guerin (BCG) or infection with $M$. lepraemurium $[8,9]$ are associated with impaired brain function and sickness behaviour. Proinflammatory cytokines produced in the periphery by activated immune cells have access to the brain, through cellular, humoral and neural routes [10], and there is evidence that they contribute in the development of neuroinflammation and neuropsychiatric symptoms such as depression and anxiety.

TB patients usually feel fear and stigma, suffer social isolation within their family and community, and generate a disillusioned and pessimistic reaction to their initial diagnosis [11]. All these changes produce depressive states that affect relationships with their friends and family. Hospitalised TB patients had mild $(22 \%)$, moderate $(38 \%)$ and severe $(8 \%)$ depression. Moreover, $15.1 \%$ of TB patients suffer from anxiety disorder $[12,13,14]$. Indeed, chronic pulmonary diseases are among the most strongly associated with psychiatric disorders such as depression and anxiety [15]. Thus, there is evidence that TB is not only a chronic infection but also a biopsychosocial disease with comorbid depression and anxiety.

Considering that the lung is a highly innervated organ and the fact that the infection by Mtb induces chronic inflammation with significant abnormalities of cell-mediated immunity [5], we hypothesized that the behavioural abnormalities exhibited during pulmonary TB could be due to chronic lung inflammation, that produces neuroinflammation with the above mentioned neuropsychiatric abnormalities. Thus, in this work, we used a well-characterised murine model of progressive pulmonary TB to study CNS inflammation and its relationship with behavioural changes, in the absence of culturable mycobacteria in the brain.

Furthermore, we found that a marked synthesis of cytokines within the hypothalamus, hippocampal formation and cerebellum that followed the kinetics of lung inflammatory response. The pattern of synthesis of these cytokines was coincident with the mentioned neuropsychiatric abnormalities, as chronically infected mice featured progressive declining in cognitive and behavioural tasks. As expected, histopathological and biochemical evidence of neuronal stress was found in these brain-specific areas. These results suggest that pulmonary TB produced a neuroinflammatory state and neuronal damage that could be associated with psychopathology.

\section{Methods}

\section{Reagents and antibodies}

The Middlebrook 7H9 and 7H10 media and the OADC (oleic acid, albumin, dextrose and catalase) were obtained from Becton-Dickinson (Detroit MI, USA). The Rneasy ${ }^{\circledR}$ Mini Kit for RNA extraction, the 
Omniscript ${ }^{\circledR}$ Reverse Transcription Kit for obtaining complementary DNA and the QuantiTectTM SYBR ${ }^{\circledR}$ for RT-PCR were purchased from Qiagen (Germantown, MD, USA). The primers of the analysed genes and pierce BCA Protein Assay kit for protein quantification were got from Invitrogen ${ }^{\mathrm{TM}}$ Thermo Fisher Scientific (Waltham, MA, USA). The standards for dopamine, serotonin, epinephrine, and norepinephrine, as well as ascorbic acid, L-cysteine, bovine serum albumin (BSA), phenylmethylsulfonyl fluoride (PMSF) and a-tubulin antibody (T9026), were obtained from Sigma Chemical Co. (St. Louis, MO, USA). HPLC grade acetonitrile, perchloric acid, and EDTA were purchased from J.T. Baker (Mexico City, Mexico).

Primary antibodies against phospho-JNK (4671), JNK (9252), phospho-p38 (9215) and p38 (9212), were purchased from Cell Signaling Technology (Danvers, MA, USA). Primary antibodies against BDNF (ab72439) was obtained from Abcam Inc. (Cambridge, MA, USA). Secondary antibodies against rabbit (711-035-152) and mouse (715-035-150) were purchased from Enzo Life Sciences (Farmingdale, NY, USA). Primary antibodies against TNFa (SC-1351), IFNy (SC-1379), IL1 $\beta$ (SC-1251), IL4 (SC-1260), iNOS (SC-651), TGF $\beta$ (SC-146) and indolamine 2-3 dioxygenase (IDO) (SC-13147) were purchased from Santa Cruz Biotechnology Inc. (Dallas, TX, USA).

Fluoro-jade B was purchased from Millipore (Bedford, MA, USA). All the other reagents were obtained from known commercial sources.

\section{Animals}

A total of 560 adult male BALB/c mice eight weeks old were obtained from the animal house facility of the National Institute of Medical Science and Nutrition Salvador Zubiran (INCMNSZ), Mexico. Mice were group-housed ( $n=5 /$ cage) and randomly divided into two groups: control (CT, $n=256)$ and infected $(H 37 R V, n=304)$. All the animals were kept in an Accredited animal holding facility maintained at a controlled temperature $\left(23 \pm 1^{\circ} \mathrm{C}\right)$ and humidity $(50 \pm 20 \%)$ under a 12:12 h light: dark cycle (lights on at 07:00 h). Food and water were provided ad libitum. All the animal experiments were done according to the guidelines of the ARRIVE and Mexican Constitution law NOM 062-Z00-1999, and approval by the Ethical Committee for Experimentation in Animals of the INCMNSZ in Mexico protocol number: PAT-186516/19-1.

\section{The experimental model of pulmonary TB}

The murine model of progressive pulmonary TB was described previously [16]. Briefly, the reference Mtb strain H37Rv was cultured in 7H9 medium with OADC enrichment. Mid-log-phase cultures were used for all experiments. Mtb were counted and stored at $-80^{\circ} \mathrm{C}$ until use. Bacterial aliquots were thawed and pulse-sonicated to remove clumps. After mice infection, the remnant of the bacterial inoculum was plated to confirm the number and viability of colony-forming units (CFU) administered to the animals. Male BALB/c mice, 8 weeks of age, were anaesthetized in a gas chamber using $0.1 \mathrm{~mL}$ per mouse of sevoflurane. A blunt stainless steel cannula with a small ball in its terminal end was inserted through the 
mouth and directed to the trachea, proper intratracheal placement of the cannula was verified by palpation of the small ball from the cannula rubbing the tracheal rings. Mice were infected through intratracheal instillation with $2.5 \times 10^{5}$ live bacilli.

Mice were maintained in a vertical position until spontaneous recovery. A total of 304 infected mice were maintained in groups of five in cages fitted with micro-isolators in a P-3 biosecurity level facility.

\section{Experimental design}

We analyzed the effects of pulmonary TB in the CNS inflammation and its relationship with behavioural changes. Following infection, mice were euthanized by exsanguination under anaesthesia at days 1, 3, 7, $14,21,28,60$ and 120 post-infection; lungs and brain were collected immediately to determine bacillary loads by colony-forming units counts (CFU). In selected areas of the brain were quantified cytokines gene and protein expression by quantitative reverse transcription-polymerase chain reaction (RT-PCR) and immunohistochemistry $(\mathrm{IHC})$, respectively, the concentrations of the neurotransmitters by highperformance liquid chromatography (HPLC) and the histopathology alterations. A total of 256 noninfected mice (CT group) received only the vehicle (saline solution) by intratracheal route following the same procedure and were used as controls. Different behavioural tests were performed during pulmonary TB. These tests include the study of sickness behaviour (locomotor activity, food intake and weight loss), anxiety-like behaviour, neurological severity score, short and long-term memory and depression-like behaviour.

\section{Determination of colony-forming units (CFU) in infected lungs and brain}

Right lungs and brains from six mice at each time point of two independent experiments were used for bacterial colony counting. Lungs and brains were homogenised with a FastPrep homogeniser (MP Biomedicals) in sterile tubes containing $1 \mathrm{ml}$ of isotonic saline solution. Four dilutions of each homogenate were spread onto duplicate plates containing Bacto Middlebrook $7 \mathrm{H} 10$ agar, enriched with OADC. Incubation time and CFU counting was at 21 days of plating [17].

\section{Expression of cytokines by RT-PCR}

Hippocampus, hypothalamus and cerebellums from six CT and infected animals at each time point were used to isolate mRNA using the Rneasy Mini Kit, according to recommendations of the manufacturer. Quality and quantity of RNA were evaluated through spectrophotometry (260/280) and on agarose gels.

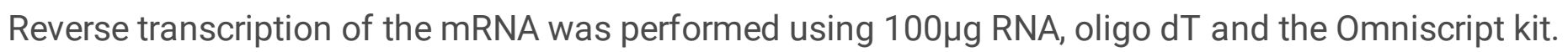
Real-time PCR was performed using the 7500 RT-PCR system (Applied Biosystems, USA) and Quantitec SYBR Green Mastermix kit (Qiagen). Standard curves of quantified and diluted PCR product, as well as 
negative controls, were included in each PCR run. Specific primers for genes encoding glyceraldehyde-3phosphate dehydrogenase (GAPDH) as housekeeping gene and for TNF-a, IFN- - , Interleukin (IL) 12, IL-10, IL-4, TGFb, iNOS and IDO were designed using the program Primer Express (Applied Biosystems, USA). The gene expression of these cytokines was determined as previously described [17]. Cycling conditions used were: initial denaturation at $95^{\circ} \mathrm{C}$ for $15 \mathrm{~min}$, followed by 40 cycles at $95^{\circ} \mathrm{C}$ for $20 \mathrm{~s}, 60^{\circ} \mathrm{C}$ for $20 \mathrm{~s}$, and $72{ }^{\circ} \mathrm{C}$ for $34 \mathrm{~s}$. Quantities of the specific mRNA in the sample were measured according to the corresponding gene-specific standard.

\section{Neurotransmitter quantification by HPLC}

The neurotransmitters quantification was previously described [9]. Briefly, the hippocampus, cerebellum and the hypothalamus of six CT and infected animals were homogenised using $400 \mu$ l of a solution containing $5 \%$ ascorbic acid, $200 \mathrm{mM}$ sodium phosphate, $2.5 \mathrm{mM}$ L-cysteine, and $2.5 \mathrm{mM}$ EDTA. Proteins were precipitated by the addition of $100 \mu \mathrm{l}$ of $0.4 \mathrm{M}$ perchloric acid, followed by incubation at $20^{\circ} \mathrm{C}$ for 20 min. Supernatants containing norepinephrine (NE), epinephrine (EP), dopamine (DA), and serotonin (5$\mathrm{HT}$ ) were collected after centrifugation at $16128 \mathrm{~g}$ for $10 \mathrm{~min}\left(4^{\circ} \mathrm{C}\right)$. NE, EP, DA, and 5-HT concentrations were determined by reversed-phase HPLC (RP-HPLC) in a system integrated by two 515 pumps (Waters ${ }^{\text {TM }}$ ), degasser AF (Waters ${ }^{\mathrm{TM}}$ ), 717 autosampler $\left(\right.$ Waters $^{\mathrm{TM}}$ ), and an X-LC ${ }^{\mathrm{TM}}$ 3120FP fluorescence detector (Jasco, Inc). Instruments were controlled by Millenium 32 software $\left(\right.$ Waters $\left.^{\mathrm{TM}}\right)$. Chromatographic runs were performed using a Jupiter C18 column ( $300 \AA$, five $\mu, 4.6 \times 250 \mathrm{~mm}$, Phenomenex $\circledast)$ at $30^{\circ} \mathrm{C}$. The column was equilibrated with the mobile phase $A$ (MPA) containing $0.1 \%$ trifluoroacetic acid. Mobile phase $B$ (MPB) containing $0.1 \%$ trifluoroacetic acid in acetonitrile was used to perform a linear gradient until reaching 20\% MPB, from min 5 to min 15 . Ten, 20\% MPB was maintained until min 20; the flow rate was $0.8 \mathrm{ml} / \mathrm{min}$. The fluorescence detector was set at gain 100 , attenuation 32 , response $20 \mathrm{~s}$, and $280 \mathrm{~nm}$ and $315 \mathrm{~nm}$ for excitation and emission, respectively. The sample injection volume was $50 \mu \mathrm{l}$. The concentration of each neurotransmitter was obtained by $\mathrm{mg}$ of protein in each sample. The concentration of total protein was obtained with the Pierce BCA protein assay kit.

\section{Behaviour tests}

In order to avoid potential habituation to the test, groups of mice were tested only once at the mentioned time points post-infection in control and infected mice. Animals were habituated to the test environment $24 \mathrm{~h}$ before that test was made. All behavioural trials were performed during the first $4 \mathrm{~h}$ of the dark phase of the light cycle.

\section{Locomotor activity}

The effect of Mtb lung infection on locomotor activity (LMA) was evaluated in mice individually positioned into a clean, novel cage similar to the household pen, but devoid of bedding or litter allowing 
the mouse to move between compartments freely. The box was divided into eight quadrants, animals were video recorded, and LMA was measured by counting the number of crossings for $10 \mathrm{~min}$.

\section{Food intake}

In order to estimate the food intake, twice a week, the amount of food given to mice was weight, and the total ingesting of food by mice was calculated as is indicated in the next formulation where $\mathrm{n}$ is the number of mice per cage. Data are expressed as $\mathrm{g} / \mathrm{mouse} /$ day.

$$
\text { Food intake }=\frac{\left[\frac{\text { Initial food }(g)-\text { final food }(g)}{\text { days }}\right]}{n}
$$

\section{Weight loss}

The weight loss of the animals infected with Mtb was estimated from day one post-infection until day 120. Each week the animals were weighted, and their loss weight recorded as a feature of sickness behaviour.

\section{Depression-like behaviour}

The tail suspension test [18] was carried out to evaluate depression-like- behaviour. Animals were suspended from the tail for $6 \mathrm{~min}$ in a tripod of $30 \mathrm{~cm}$ height, and their activity was recorded, focusing at the moment when the animal stopped moving. The time that the animal remains both mobile and immobile for 6 min was recorded.

\section{Anxiety-like behaviour}

The anxiety-like behaviour was evaluated studying the Stretched attend posture (forward elongation of the body) [19]. In this test was used a large circular platform raised at 35-40 cm from the floor, and a small circular awning fastened by the central pillar was placed at $4 \mathrm{~cm}$. Each mouse was located in the covered area of the platform and filmed for $5 \mathrm{~min}$. The number of stretched attend postures was counted as a measure of anxiety-like behaviour.

\section{Neurological Severity Score (NSS)}

Motor function and reflexes of the infected mice were evaluated using a neurological severity score [20]. It was valued regarding absent (0) or present (1), except for the hypomobility, motor impairment and balance that is rated as weak (1), moderate (2) or strong (3). The maximum rating of 31 (indicating neurological damage). Usual, from 3 to 6 ( For more details, see Additional File 1).

\section{Evaluation of learning and memory.}


Memory and learning after pulmonary infection with Mtb were assessed with the Object Recognition Test [21]. With this test, we evaluated short-term and long-term memory. In a first habituation phase, we placed the animal in the open field without any object for $10 \mathrm{~min}$ to become familiar with the environment. At 24 $h$, two identical objects (objects $A$ ) were placed in different positions, and the animal was left inside the box for $3 \mathrm{~min}$. In the next phase, short-term memory was measured, for this we positioned an object $A$ (familiar object) and placed a new object in the other position (object B), the interactions with both objects (the animal sniffs or touches the object with the front legs) were counted during $3 \mathrm{~min}$. After 24 $h$, the long-term memory was measured, for which object $B$ was changed to a novel object (C), and the same procedure was followed. The results are presented as the discrimination ratio, which is the difference in interactions expressed as a proportion of the total interactions with the two objects in both tasks.

\section{Preparation of brain tissue for histological analysis}

Four mice per selected time point were anaesthetised with sodium pentobarbital ( $100 \mathrm{mg} / \mathrm{kg}$, i.p.) and immediately perfused transcardially with isotonic saline solution, followed by cold $4 \%$ paraformaldehyde solution diluted with Sorensen's phosphate buffer $(0.133 \mathrm{M}, \mathrm{pH} 7.2)$. Brains were removed and postfixed in $4 \%$ paraformaldehyde for 24 hours and embedded in paraffin. Coronal sections $4 \mu \mathrm{m}$ thick mounted on glass slides, deparaffinised, and stained with hematoxylin and eosin. For quantification of tissue damage, five fields of regions CA1, CA2, CA3, and dentate gyrus (DG) of the hippocampus were analysed under a light microscope Q-win Leica 500 to estimate the number of neurons showing morphological damage (cell shrinking, condensed hyperchromatic nucleus, basophilic cytoplasmic material disappearance, or cytoplasmic vacuoles). The results are presented as a percentage of injured neurons in hippocampal regions.

\section{Immunohistochemistry (IHC) analysis}

The same brain tissue samples for the histological study were used for IHC. Coronal sections ( $4 \mu \mathrm{m}$ thick) were mounted on poly-L-lysine-coated slides and then deparaffinized to detect activated microglia (IBA-1, 1:3000), gliosis (Glial fibrillary acidic protein GFAP, 1:3000), TNFa (1:250), IFNg (1:250), IL4 (1:250), and TGFb (1:200). After deparaffination, tissue sections were blocked for the unspecific activity of endogenous peroxidase with $3 \%$ hydrogen peroxide solution and methanol by $10 \mathrm{~min}$. Subsequently, sections were incubated with each antibody and then incubated with goat anti-rabbit IgG-peroxidase diluted 1:500 for 30 min at room temperature. After extensive washings with PBS, the bound antibodies were detected with diaminobenzidine and counterstained with hematoxylin.

\section{Localization of neuronal damage in the hippocampus by Fluoro-jade B (FJ-B) staining}


FJ-B staining is a useful technique to determine damaged neurons that are still alive (degenerating neurons) and was done according to the published protocol [22]. Briefly, tissue sections $8 \mu \mathrm{m}$ thick were deparaffinised and immersed in a solution containing $1 \% \mathrm{NaOH}$ in $80 \%$ ethanol for $5 \mathrm{~min}$, followed by 2 min in $70 \%$ ethanol and 2 min in distilled water. Afterwards, the sections were immersed in a $0.06 \%$ $\mathrm{KMnO} 4$ solution for 10 min under moderate moving and then cleaned in distilled water for $2 \mathrm{~min}$. Tissue sections were stained with $0.0004 \%$ FJ-B solution for 20 min and washed three times with distilled water. The slides were dried in an oven at $50^{\circ} \mathrm{C}$ for $15 \mathrm{~min}$ and cleared with xylene before coverslipping. Two random fields were selected in each mouse from the different experimental groups. Results are expressed as the number of FJ-B positive cells per field.

\section{Study of the activation of MAPK signalling by Western blot assays}

In order to study if the mitogen activated protein kinases (MAPK) are involved in the cellular damage, the expression of JNK and p38 activated (phosphorylated) and non-activated was determined by westernblot, as well as the levels of the neurotrophin brain derived nerve growth factor (BDNF) using the same technique. The hippocampus, hypothalamus and cerebellum were dissected quickly and homogenized in $500 \mathrm{~mL}$ of lysis buffer pH 7.9 (containing $20 \mathrm{mM}$ Tris $\mathrm{HCl}, 30 \mathrm{mM} \mathrm{NaCl}, 0.5 \mathrm{mM}$ sucrose, $1 \mathrm{mg} / \mathrm{mL}$ leupeptin, $1 \mathrm{mg} / \mathrm{mL}$ aprotinin, $1 \mathrm{mg} / \mathrm{mL}$ pepstatin, $1 \mathrm{mg} / \mathrm{mL}$ PMSF and $1 \mathrm{mg} / \mathrm{mL}$ phosphatases inhibiting cocktail) and centrifuged at $20,800 \mathrm{~g}$ for $30 \mathrm{~min}$ at $4^{\circ} \mathrm{C}$. The supernatants were used to determine the protein quantity using Lowry's technique and in the western blots assay. Briefly, $50 \mu \mathrm{g}$ of the total lysate of protein were loaded and separated in $10 \%$ or $12 \%$ SDS polyacrylamide gel electrophoresis and transferred to polyvinylidene fluoride (PVDF) membranes (Millipore, Bedford, MA, USA). Membranes were blocked using $5 \% \mathrm{BSA}$ for $2 \mathrm{~h}$ at room temperature with slight agitation. Blots were then incubated with anti-phospho-JNK $(1: 1,000)$ at room temperature for two hours, anti-JNK $(1: 1,000)$, anti-phosphop38 $(1: 1,000)$, anti-p38 $(1: 1,000)$, anti-BDNF $(1: 1,000)$, or anti- $\beta$-tubulin $(1: 8,000)$ at $4^{\circ} \mathrm{C}$ for overnight. Membranes were washed three times (10 min) with TBS plus $0.1 \%$ Tween (TBS-T). A horseradish peroxidase-conjugated secondary polyclonal antibody anti-rabbit $(1: 10,000)$ and anti-mouse $(1: 10,000)$ was then added for $2 \mathrm{~h}$ and after extensive washing. Bands were detected using the enhanced chemiluminescence of detection system (ECL, Amersham Pharmacia Biotech, USA). The membranes were washed with stripping solution (containing $0.2 \mathrm{M}$ glycine, $0.1 \%$ SDS and $1 \%$ Tween 20, pH 2.2) for the detection of two or more proteins. The chemiluminescence imaging system Fusion Solo $S$ (Eberhardzell, Biberach, Germany) was used. Areas values were obtained from the relationship between the optical densities (OD) of each band. Area values of each group were standardised to the area value of the control group (value= 1). Data are expressed as the OD ratio using ImageJ software.

\section{Assessment of blood-brain barrier dysfunction using Evans Blue staining}


We analysed the blood-brain barrier (BBB) dysfunction, according to a previously published protocol [23]. Briefly, mice were anaesthetised with sodium pentobarbital (100 mg/kg, i.p.) and injected with Evans blue (EB) $(2 \%, 2 \mathrm{ml} / \mathrm{kg})$ in the caudal vein $3 \mathrm{~h}$ before perfusion. Mice were perfused transcardially with isotonic saline solution and directly beheaded, stripping brain tissue on ice. Each brain was weighed and then homogenised with a FastPrep homogeniser (MP Biomedicals) in $0.25 \mathrm{ml}$ of $100 \%$ TCA and $0.75 \mathrm{ml}$ of PBS solution. Samples were cooled overnight at $4{ }^{\circ} \mathrm{C}$ and then centrifuged for $30 \mathrm{~min}$ at $1,000 \times \mathrm{g}$ at $4{ }^{\circ} \mathrm{C}$. The EB in the supernatants of $100 \mu$ of each sample was then measured at $620 \mathrm{~nm}$ using a 96-well plate reader. All measurements were within the range of detection established by a standard curve. The dye concentration was considered as the ratio of absorbance relative to the amount of tissue.

\section{Statistical analysis}

Data are expressed as the mean \pm standard error of the mean (SEM) of two independent experiments. The statistical analysis was performed using Prism software version 7.0 (GraphPad, San Diego, CA, USA). For the survival curve, the Log-Rank and Kaplan-Meier test was applied. Cytokine expression by RT-PCR, behavioural assays, loss of body weight, food intake and neurotransmitter levels were analysed by Two Way ANOVA followed by Sidak multiple comparison tests (comparison of each group against the control). A value of $p £ 0.05$ was considered statistically significant.

\section{Results}

\section{Progressive pulmonary tuberculosis is not accompanied by a brain infection.}

Brains collected from mice infected with Mtb H37RV did not show bacillary growth colonies in any day of infection. These results indicate that a brain infection does not accompany progressive pulmonary tuberculosis. In contrast, the bacillary loads in lung increased progressively after 3 weeks of infection and until day 120 , with an average bacillary load of 30 million CFU, when the main number of animals started to die after 2 months of infection (see Additional File 2).

\section{Cytokine synthesis is increased in selected areas of the brain during pulmonary TB}

Even in the absence of cultivable bacteria in the brain, substantial changes in the levels of cytokines expression were seen in the hippocampus, hypothalamus and cerebellum of tuberculous mice. These three anatomical areas are critical in neural processes, such as motor function, cognition, behaviour and memory, so the modification in the levels of cytokines expression in these areas could be related with behavioural alterations. 
In the hypothalamus, a decrease of gene expression of proinflammatory (TNFa and IL-12), antiinflammatory (IL-4) cytokines (Fig. 1), as well as TGF $\beta$ and the enzymes IDO and iNOS (see Additional File 3) was seen during the early phase (1-14 days) of the infection. Then, a significant increase in the expression of these cytokines was detected since day 21 post-infection, when the peak of maximal immune protection is seen in this murine model. In contrast, the expression of IFNy was significantly decreased in the tuberculous animals in all the studied time points in comparison with the non-infected control animals (Fig. 1).

The hippocampus and cerebellum showed a highly significant increase in the expression of the proinflammatory (TNFa, IFNy, IL12) and the anti-inflammatory cytokines (IL4) (Fig. 1), as well as TGF $\beta$ and the enzymes iNOS and IDO since day one post-infection. The anti-inflammatory cytokine IL10 was only expressed in the cerebellum of the infected animals from day one after infection in small amounts, increasing significantly from day 14 of infection (see Additional File 3).

Regarding the immunohistochemistry study, control non-infected mice showed slight cytokines immunostaining in the brain selected areas (not shown); in contrast, infected animals showed a marked increase in the positivity of the immunoreaction for IFNy, TNFa, TGF $\beta$ and IL4 in numerous neurons and glial cells in the hippocampus, mostly from the CA3 region and the dentate gyrus. Except for IFNy, the same pattern was seen in neurons and glial cells located in the paraventricular nucleus of the hypothalamus. Purkinje cells from the cerebellum cortex showed intense immunoreactivity to IL4, IL1 $\beta$, IFNy and TNFa. Interestingly, the endothelium from numerous capillaries and activated microglia in the same area showed strong immunostaining to TGF- $\beta$. Epithelial cells and stroma of the choroid plexus also showed strong immunostaining to these cytokines. Tuberculous animals exhibited numerous cells with strong immunoreactivity to GFAP and Iba-1, indicating gliosis and activated microglia, respectively, after 60 days of infection (Fig. 2).

\section{There are important changes in the levels of neurotransmitters during pulmonary TB}

The immune and the nervous systems maintain constant communication through cytokines and neurotransmitters, which bind to specific receptors expressed by specialised cells of both systems. An essential physiological process of this neuroimmunological communication is the response to stress, such as induced by a severe infectious disease like TB, whose dominant response occurs in the noradrenergic and adrenergic neurons; as well as the dopaminergic and serotonergic systems. The anatomical areas where these responses are integrated are the locus coeruleus, nucleus tractus solitarius (NTS), cortex, hippocampus, cerebellum and hypothalamus [24]. In the present work, the neurotransmitters NE, DA, EP and 5-HT were measured in the hippocampus, hypothalamus and cerebellum of mice with pulmonary TB without cultivable bacteria in the brain. 
In contrast with control animals, mice with pulmonary TB showed a significant decrease of NE, DA, EP and $5-\mathrm{HT}$ in the hypothalamus and cerebellum since day one of infection, being more pronounced at days 60 and 120 post-infection, when late progressive disease with high bacilli burdens and extensive pneumonia was observed. In the hippocampus, there was also a decrease in the concentration of DA and NE during pulmonary TB. At the same time, EP showed a significant increase since day one of infection, at days 21 when there is the maximal immune protection, and at day 28 post-infection when starting the progressive phase of the disease. Interestingly, the concentration of NE was significantly lower at very late disease, at day 120 of infection when the maximal bacillary burdens and pulmonary inflammation were seen (Fig. 3).

\section{Pulmonary tuberculosis is associated with diverse behavioural abnormalities}

Mice infected with Mtb H37RV showed signs of "sickness behaviour", such as body weight decrease, which started after one-month post-infection when the progressive phase of the disease started and was significant after 3 and 4 months of infection. Another feature of sickness behaviour is the decrease of locomotor activity, which was observed during the whole course of the disease being higher during early infection from day 1 to 21 . We also observed that infected animals decreased food intake, an essential characteristic of sickness behaviour (Fig. 4 A-C).

There was a significant increase in the time of immobility at the tail suspension test in the group of tuberculous animals indicative to depression-like behaviour, being the highest during late progressive TB at day 60 and 120 of infection. After 2 weeks of infection, there was a significant rise in the number of stretches attend postures that are related to anxiety-like behaviour (Fig. 4 D-E)

We also evaluated the neurological outcome (NSS). Infected animals presented a higher NSS, so the infection impaired motor activity and reflexes, since day 7 until day 120 post-infection (Fig. 4F), similar to the depressive-like behaviour.

Finally, there was a significant impairment in the short and long-term memory in infected mice, which was more evident after 14 days of infection than in the first days of infection (Fig. 4 G-H).

\section{Pulmonary tuberculosis affected components of the MAPK pathway, increasing the activation of p38 and JNK and decreasing BNDF levels.}

Most cellular responses to extracellular stimuli are regulated by kinase and phosphatase cascades. One of the most critical kinase families is the MAPK that comprises: ERK 1/2, JNK, and p38 [25] with different activities, the JNK family participates in various physiological and pathological processes, including the 
expression of inflammatory molecules, such as TNFa, IL-2, E selectin and matrix metalloproteinases [26]. In the CNS, JNKs are involved in diverse neuroinflammatory diseases such as Parkinson's disease, Alzheimer's disease and kainate-induced excitotoxicity. JNKs also generate neurodegeneration by the withdrawal of trophic molecules [27]. Another significant kinase of MAPK is p38, a serine kinase that plays a central role in many inflammatory responses including oxidative stress induced by endotoxin and proinflammatory cytokines, such as TNFa and IL-1b [28].

During late progressive TB, a significant increase of p38 activation in the hippocampus at 28 days, and in the hypothalamus at 28- and 120-days post-infection. In the cerebellum, no significant changes were observed at any time. JNK activation was observed in the hippocampus at 28 days and in the hypothalamus at 120 days post-infection. No significant changes were found in the cerebellum at any time of treatment (Fig. 5).

BDNF is a member of the neurotrophic factor family that plays a crucial role in regulating survival, growth, and maintenance of neurons. Besides, BDNF plays a role in learning and memory, and its decreased production is a pathogenetic factor of major depression [29]. BDNF concentrations were significantly decreased in the hippocampus and the hypothalamus at 60 days and in the cerebellum at 120 days post-infection (Fig. 5).

\section{Pulmonary tuberculosis induces neuronal damage during early infection and neural death in the hippocampus and blood-brain barrier dysfunction during progressive late disease}

The FJ-B staining to detected still alive damaged neurons showed positive cells in the hippocampus (CA3) since day 1 to 14 after infection (Fig. 6 A-B). No positive cells to FJ-B were seen in later days (data not shown). This result indicates the presence of neuronal damage in the early stage of infection.

The histological damage in the hippocampus of tuberculous animals was determined according to several parameters, particularly those indicative of cell death such as basophilic pyknotic nuclei, acidophilic cytoplasm, and fragmented nuclei in regions CA1, CA2, CA3, and DG. The general morphology of hippocampus in the day one post-infection was well preserved, with neurons of typical characteristics. By day 60 post-infection, neurons with pyknotic nuclei and acidophilic cytoplasm were observed in the four regions analysed being higher in the DG. By day 120 , the number of pyknotic-acidophilic neurons increased in the four studied areas (Fig. 6 C-D).

Inflammation-induced damage to the BBB, which was evaluated by BBB dysfunction in mice with pulmonary TB. There was a significant increase in the BBB permeability at days 60 and 120 postinfection (Fig. 6 E). 


\section{Discussion}

There has been consistent information that under physiological conditions, low production of inflammatory cytokines, such as TNF- $\alpha$ and IL-1 $\beta$, are involved in several essential brain processes such as synaptic remodelling, neurogenesis and long-term potentiation [30]. Thus, these immune mediators have an essential role in neuronal integrity, and interestingly, their overproduction can exert profound effects on neural circuits that ultimately affect behaviour [31]. This situation has been observed in diverse diseases that induce chronic peripheral inflammation, such as arthritis, diabetes mellitus, obesity and lupus erythematosus, which affect the nervous system producing behavioural abnormalities, impairment of learning and memory in coexistence with depression [7]. TB is an infectious-contagious disease that affects mainly the lungs producing thoughtful alterations in the immune system and characteristically induce chronic inflammation. The present work demonstrated for the first time that neuroinflammation and behaviour abnormalities are produced during experimental pulmonary TB in the absence of cerebral infection, suggesting that neurological abnormalities, even neuronal death, could be caused by the intense and chronic pulmonary inflammation.

In mice and humans, mycobacterial pulmonary infection is mainly controlled by activated macrophages and Th-1 lymphocytes [32]. Pro-inflammatory cytokines such as IFN- $y$ and IL-12 are essential to this process because they induce differentiation and activation of Th-1 lymphocytes, which stimulate the activation of macrophages that produce TNF- $a$ and nitric oxide after the induction of iNOS. Nitric oxide is essential for killing intracellular mycobacteria [33]. This protective activity fails if there is a marked release of Th-2 type cytokines [34, 35]. This interplay of cytokines is seen in our BALB/c model of progressive pulmonary TB induced by intratracheal inoculation of a high dose of the reference Mtb strain H37Rv $[16,36]$. In this model, at day 1 and 3 after infection, there is a rapid and high production of proinflammatory cytokines, such as TNFa and IL-1 $\beta$ by activated macrophages located in the alveolarcapillary interstitium and alveolar lumen. After 2 weeks of infection, granulomas started their formation in co-existence with high expression of IFN- $y$, TNF-a, IL-12 and IL-17 that permit efficient control of bacilli growth. Then, after 1 month of infection, this Th- 1 cytokine pattern decreases, and Th- 2 cells emerge in coexistence with a high burden of bacilli and progressive pneumonia, which finally cause animals death. These pro-inflammatory cytokines produced and released by activated peripheral monocytes and macrophages in the infected lung can circulate and access to the brain through leaking regions of the $\mathrm{BBB}$, such as the choroid plexus and circumventricular organs (CVOs) [37, 38]. Another way to induce neuroinflammation is the neural route, which involves the activation of the sensory fibres of the afferent vagus nerve that informs the brain of the peripheral inflammatory process [39]. The vagus nerve widely innervates the lung, and diverse immunological stimuli activate vagal afferents directly or indirectly. Direct stimulation occurs through receptors located in the nerve terminations to cytokines released by fibroblasts, endothelial cells, dendritic cells, macrophages and other immune cells, and by the binding of microbial pathogen-associated molecular patterns (PAMPS) to their receptors, such as TLRs that also exist in the vagus nerve. The sensory afferences conducted by this nerve transmit information to diverse brain areas through an initial activation of the tractus solitarius nucleus (NTS) and area postrema [10]. The high production of peripheral inflammatory cytokines or activated immune cells, as well as the vagus 
afferent nerve stimulation, can modify the tone of neuronal networks and induce neuroinflammation by inducing resident production of cytokines [40]. Our RT-PCR and immunohistochemistry studies clearly showed this high production of inflammatory cytokines in the brain parenchyma. Cytokines in the brain are produced primarily by microglia, but can also be produced by astrocytes or oligodendrocytes, and as our immunohistochemistry study shows by neurons and even by choroidal epithelial cells. Reactive astrocytes can also play detrimental roles during injury or disease through gain of atypical effects, such as overproduction of reactive oxygen species (ROS) or specific inflammatory cytokines [41]. Moreover, the overproduction of proinflammatory cytokines in the brain tissue activates endothelial cells and perivascular macrophages, that express the enzymes iNOS, cyclooxygenase-2 (COX-2) and IDO [42, 43], which respectively produce nitric oxide, prostaglandins and degrades tryptophan resulting in a decrease of available tryptophan and increase of kynurenine. This decrease in tryptophan leads to the reduction of serotonergic neurotransmission and hence depression in humans and the development of depression-like behaviour in mice [44]. Indeed, we observed a significant decrease in the concentration of 5-HT, as well as DA, NE and EP in the hypothalamus, hippocampus and cerebellum of infected animals, which was more accentuated during the late progressive phase of TB in coexistence with extensive chronic pulmonary inflammation. Inflammation should lead to even more persisting alterations in the neurotransmitter function and development of depression and other psychiatric conditions [45], such as decrease of early and late memory [46]. NE partly regulates initial memory and participates in diverse cognitive, affective and behavioural functions [47], it also has anti-inflammatory and antioxidant functions in astrocytes and microglia [48]. However, excessive stress such as produced during advanced pulmonary TB, produced NE decrease affecting the process of memory acquisition [46], and contribute to depression, attention deficit, social isolation and difficulties of concentration [49]. Regarding DA, low concentrations of this neurotransmitter, particularly in basal ganglia has been related to anhedonia, fatigue, and psychomotor slowing. Experimental evidence showed that high production of cytokines, such as IFNy, influence the synthesis, recapture and secretion of DA [50] in association with high production of NO that induced oxidative cellular damage [51].

A similar response to that observed in this study with TB about the high expression of proinflammatory cytokines in the cerebellum, hippocampus and hypothalamus was also produced in animals infected with Plasmodium falciparum [52] or coinfected with Toxocara canis and Toxoplasma gondii [53, 54]. Diverse experiments showed that the administration of the non-virulent BCG in CD1 mice, C57BL / 6J mice and BALB / c mice causes sickness behaviour $[55,56,57,58]$, but due that BCG is a non-virulent bacteria, there is rapid and efficient bacterial elimination with limited inflammation. While pulmonary infection produced by virulent mycobacteria, which produce progressive disease with chronic pulmonary inflammation, such as our TB model, induced chronic neuroinflammation and behaviour abnormalities. Thus, our behavioural tests in tuberculous BALB/c mice showed a significant decrease of motor activity and food intake during early Mtb infection, which is a characteristic of sickness behaviour, being at day 21 when the lowest motor activity was observed. Sickness behaviour was seen in coexistence with high production of TNFa and IL-1 $\beta$ by macrophages from mature pulmonary granulomas that efficiently control bacillary growth [16]. Then, after 1 month of infection there is the highest increase of 
proinflammatory cytokines in the hippocampus that should participate in the damage of short- and longterm memory, by inducing neuronal death, such as was seen in the C3 region of the hippocampus of tuberculous mice.

The immunological and neurochemical changes that we observed in the tuberculous animals coincided particularly well during late progressive disease with neurodegeneration, neuronal death and the increase of permeability of the BBB. Thus, it seems that neuroinflammation produces neuronal oxidative damage during early disease and neuronal death during progressive pulmonary disease. To study the mechanism of cell damage induced by neuroinflammation, we explored the signalling pathway of the MAPKs system, determining during late disease, at days 28, 60 and 120 after infection, the expression of p38 and JNK, which are critical regulators of the MAPK system related to inflammatory and stress processes.

The MAPK family is constituted by the extracellular signal-regulated kinase (ERK), p38 and c-Jun NH2terminal kinase (JNK). In vitro studies have associated JNK and p38 activation (phosphorylated) as promoters of cell death under in vitro excitotoxic conditions $[59,60]$. We found significant overexpression of p38 and JNK in hippocampus, hypothalamus and cerebellum during late progressive pulmonary TB, suggesting that this intracellular system is contributing to neuronal damage and death probably by oxidative stress under these conditions. Furthermore, the MAPK signalling pathway can also participate in the production of pro-inflammatory cytokines, considering that mycobacteria induce the phosphorylation of JNK, p38 and ERK that contribute to the production of TNFa, IL-1 $\beta$, and MCP-1 [61]. Another critical aspect of the activation of this system is the decrease of extracellular signals, such as the neurotrophin BDNF that promotes cell survival $[62,63]$ and anti-inflammation, by suppressing the production of pro-inflammatory mediators via the inhibition of NF-KB [64]. In the present study, a significant decrease of BDNF was seen after 60 and 120 days of infection in hippocampus and cerebellum, suggesting that the recovering of neuronal damage is also affected. However, some protective and anti-inflammatory activity should exist and exacerbate by anti-inflammatory cytokines, such as IL-4 and TGF $\beta$ that were highly expressed by glial cells, neurons and endothelial cells. Indeed, both IFNy and IL-4 have neuroprotective activities after injury, supporting neuronal survival $[65,66]$, being Th-2 cells more efficient to confer neuroprotection than Th-1 cells [67]. Interestingly, CNS injury is associated with systemic Th-2 shift [68], which may be necessary for down-regulating the Th-1 response and facilitating the progression of pulmonary TB. Another significant neuroprotective and antiinflammatory cytokine is TGF- $\beta$ [69], that was explicitly detected in endothelial cells and microglia, as well as IL-10, which was only found in the cerebellum.

\section{Conclusion}

In summary, these experimental findings suggest that the existence of peripheral inflammation with systemic release of inflammatory cytokines and afferent nerve stimulus from the lung during pulmonary infection with $M t b$ leads to persisting neuroinflammation, that produces thoughtful effects in the brain and behaviour as a result of communication between the periphery and brain. Brain alterations included the high production of different cytokines in the hypothalamus, hippocampus and cerebellum, changes in 
the levels of the neurotransmitters NE, EP, 5-HT and DA, neurodegeneration, neuronal cell death and BBB dysfunction. The intracellular molecular mechanisms could be associated with the increase in the levels of JNK and p38 activated and to the decline in the BDNF levels (Fig. 7). Altogether, these results could explain the behavioural abnormalities present in pulmonary TB and evoke the interest and necessity to control neuroinflammation in TB patients with neuropsychiatric abnormalities, that it seems not only produced by psychological factors.

\section{Abbreviations}

Analysis of variance (ANOVA), Bacillus Calmette-Guerin (BCG), Biosafety laboratory-level three (BSL-3), Blood-brain barrier (BBB), Bovine serum albumin (BSA), Central Nervous System (CNS), Circumventricular organs (CVOs), c-Jun NH2-terminal kinase (JNK), Colony Forming Units (CFU), Cyclooxygenase-2 (COX-2), Dentate gyrus (DG), Dopamine (DA), Epinephrine (EP), Ethylenediaminetetraacetic acid (EDTA), Evans blue (EB), Extracellular signal-regulated kinase (ERK), Fluoro-jade B (FJ-B), Glial fibrillary acidic protein (GFAP), Glyceraldehyde-3-phosphate dehydrogenase (GAPDH), High-performance liquid chromatography (HPLC), Hypothalamic-pituitary-adrenal axis (HPA), Immunohistochemistry (IHC), Indolamine 2,3dioxygenase (IDO), Induced nitric oxide synthase (iNOS), Interferon-gamma (IFNY), Interleukin (IL), Intraperitoneal (i.p.) lonized calcium-binding adaptor molecule 1 (IBA-1), Locomotor activity (LMA), Mitogen-activated protein kinases (MAPK), Monocyte Chemoattractant Protein-1 (MCP-1), Mycobacterium tuberculosis (Mtb), Neurological Severity Score (NSS), Neurotrophin brain derived nerve growth factor (BDNF), Norepinephrine (NE), Nuclear factor kappa B (NFKB), Nucleus tractus solitarius (NTS), Oleic acid, albumin, dextrose and catalase (OADC), Optical densities (OD), Pathogen-associated molecular patterns (PAMPS), Phenylmethylsulfonyl fluoride (PMSF), Pulmonary Tuberculosis (TB), Reverse transcription-polymerase chain reaction (RT PCR), Reversed-phase HPLC (RP-HPLC), Serotonin (5-HT), Standard Error of the Mean (SEM), Stretched attend posture (SAP), Sympathetic nervous system (SNS), Toll like Receptor (TLR), Transforming growth factor type-Beta (TGF $\beta$ ), Tumor necrosis factor-alpha (TNFa), Type 1 cooperating lymphocytes (Th1), Type 2 Cooperating lymphocytes (Th2).

\section{Declarations}

\section{Acknowledgements}

We would like to thank Dr Vasti Lozano Ordaz, and Biologist María Fernanda Arce Aceves for contributing to mice euthanize.

\section{Authors' contributions}

JVLE was involved in research experimentation, completion of statistical analysis, and writing of the manuscript. BMC, EBV, GPS, LP, DME, JBP and MOLT assisted with experimentation. RASM, MAZ and PDM contributed to research experimentation and assisted in editing the manuscript. RHP directed all 
aspects of this research project, including the experimental design, research experimentation, completion of statistical analysis, and writing of the manuscript. All authors read and approved the final manuscript.

\section{Funding}

This work was supported by CONACYT (Grant No. 2015-257390-M to BMC). Jacqueline Viridiana Lara Espinosa is a doctoral student from Programa de Doctorado en Ciencias Bioquimicas, Universidad Nacional Autónoma de México (UNAM) and received fellowship from CONACYT (CVU 630838).

\section{Availability of data and materials}

The datasets used and analysed during the current study are available from the corresponding author on reasonable request.

\section{Ethics approval and consent to participate}

All the animal experiments were done according to the guidelines of the ARRIVE and Mexican Constitution law NOM 062-Z00-1999, and approval by the Ethical Committee for Experimentation in Animals of the INCMNSZ in Mexico (protocol number: PAT-1865-16/19-1).

\section{Consent for publication}

Not applicable

\section{Competing interests}

The authors of this manuscript declare that there are no actual or potential conflicts of interest. The authors affirm that there are no financial, personal or other relationships with other people or organizations that have inappropriately influenced or biased their research.

\section{Author Details}

${ }^{1}$ Sección de Patología Experimental, Instituto Nacional de Ciencias Médicas y Nutrición Salvador Zubirán, CDMX 14080, México. ${ }^{2}$ Departamento de Psicoinmunologia, Instituto Nacional de Psiquiatria Ramón de la Fuente, CDMX 14370, México. ${ }^{3}$ Laboratorio de Patología Vascular Cerebral, Instituto Nacional de Neurología y Neurocirugía Manuel Velasco Suárez, CDMX 14269, México. ${ }^{4}$ Laboratorio de Neuropatología Molecular. Instituto de Fisiología Celular, Universidad Nacional Autónoma de México, CDMX 04510, México.

\section{References}

1. Pai M, Behr MA, Dowdy D, Dheda K, Divangahi M, Boehme CC, et al. Tuberculosis. Nat Rev Dis Prim. $2016 ; 2$. 
2. Rey A, Mahuad CV, Bozza VV, Bogue C, Farroni MA, Bay ML, et al. Endocrine and cytokine responses in humans with pulmonary tuberculosis. Brain Behav Immun. 2007;21:171-9.

3. Rook GAW. Endocrine and cytokine responses in humans with pulmonary tuberculosis. Brain Behav Immun. 2007;21:169-70.

4. Hernandez-Pando R, Orozco H, Honour J, Silva P, Leyva R, Rook GAW. Adrenal changes in murine pulmonary tuberculosis; a clue to pathogenesis? FEMS Immunol Med Microbiol. 1995;12:63-72.

5. Barrios-Payán J, Revuelta A, Mata-Espinosa D, Marquina-Castillo B, Villanueva EB, Gutiérrez MEH, et al. The contribution of the sympathetic nervous system to the immunopathology of experimental pulmonary tuberculosis. J Neuroimmunol. 2016;298:98-105.

6. Zetter M, Barrios-Payán J, Mata-Espinosa D, Marquina-Castillo B, Quintanar-Stephano A, HernándezPando R. Involvement of vasopressin in the pathogenesis of pulmonary tuberculosis: A new therapeutic target? Front Endocrinol (Lausanne). 2019;10:1-11.

7. Castanon N, Lasselin J, Capuron L. Neuropsychiatric comorbidity in obesity: Role of inflammatory processes. Front Endocrinol (Lausanne). 2014;5:1-10.

8. Platt B, Schulenberg J, Klee N, Nizami M, Clark JA. A depressive phenotype induced by Bacille Calmette Guérin in "susceptible” animals: Sensitivity to antidepressants. Psychopharmacology. 2013;226:501-13.

9. Becerril-Villanueva E, Ponce-Regalado MD, Pérez-Sánchez G, Salazar-Juárez A, Arreola R, ÁlvarezSánchez ME, et al. Chronic infection with Mycobacterium lepraemurium induces alterations in the hippocampus associated with memory loss. Sci Rep. 2018;8:1-12.

10. Capuron L, Miller AH. Immune system to brain signaling: Neuropsychopharmacological implications. Pharmacol Ther. 2011;130:226-38.

11. Martens WH. A review of physical and mental health in homeless persons. Public Health Reviews. 2001;29(1):13-33.

12. Long NH, Johansson E, Diwan VK, Winkvist A. Fear and social isolation as consequences of tuberculosis in Vietnam: A gender analysis. Health Policy. 2001;58:69-81.

13. Westaway MS, Wolmarans L. Depression and self-esteem: Rapid screening for depression in black, low literacy, hospitalized tuberculosis patients. Soc Sci Med. 1992;35:1311-5.

14. Aghanwa HS, Erhabor GE. Demographic/socioeconomic factors in mental disorders associated with tuberculosis in southwest Nigeria. J Psychosom Res. 1998;45:353-60.

15. Wells KB, Golding JM, Burnam MA. Psychiatric disorder in a sample of the general population with and without chronic medical conditions. Am J Psychiatry. 1988;145:976-81.

16. Hernández-Pando R, Orozcoe H, Sampieri A, Pavón L, Velasquillo C, Larriva-Sahd J, et al. Correlation between the kinetics of Th1, Th2 cells and pathology in a murine model of experimental pulmonary tuberculosis. Immunology. 1996;89:26-33.

17. Hernandez Pando R, Aguilar D, Cohen I, Guerrero M, Ribon W, Acosta P, et al. Specific bacterial genotypes of Mycobacterium tuberculosis cause extensive dissemination and brain infection in an 
experimental model. Tuberculosis Elsevier Ltd. 2010;90:268-77.

18. Nestler EJ, Hyman SE. Animal models of neuropsychiatric disorders. Nat Neurosci. 2010;13:1161-9.

19. Kaesermann HP. Stretched attend posture, a non-social form of ambivalence, is sensitive to a conflict-reducing drug action. Psychopharmacology. 1986;89:31-7.

20. Stahel PF, Shohami E, Younis FM, Kariya K, Otto VI, Lenzlinger PM, et al. Experimental closed head injury: Analysis of neurological outcome, blood-brain barrier dysfunction, intracranial neutrophil infiltration, and neuronal cell death in mice deficient in genes for pro-inflammatory cytokines. J Cereb Blood Flow Metab. 2000;20:369-80.

21. Ennaceur A, Neave N, Aggleton JP. Spontaneous object recognition and object location memory in rats: The effects of lesions in the cingulate cortices, the medial prefrontal cortex, the cingulum bundle and the fornix. Exp Brain Res. 1997;113:509-19.

22. Schmued LC, Hopkins KJ. Fluoro-Jade B: A high affinity fluorescent marker for the localization of neuronal degeneration. Brain Res. 2000;874:123-30.

23. Yang J, Jiang Y, Guo Y. Measuring Blood-brain-barrier Permeability Using Evans Blue in Mice. Bioprotocol. 2015; 5(15): e1548.

24. Dunn AJ, Wang J, Ando T. Effects of cytokines on cerebral neurotransmission: Comparison with the effects of stress. Adv Exp Med Biol. 1999;461:117-27.

25. Koistinaho M, Koistinaho J. Role of $\mathrm{p38}$ and p44/42 mitogen-activated protein kinases in microglia. Glia. 2002;40:175-83.

26. Huang P, Han J, Hui L. MAPK signaling in inflammation-associated cancer development. Protein Cell. 2010;1:218-26.

27. Waetzig V, Czeloth K, Hidding U, Mielke K, Kanzow M, Brecht S, et al. c-Jun N-terminal kinases (JNKs) mediate pro-inflammatory actions of microglia. Glia. 2005;50:235-46.

28. Schieven G. The Biology of p38 Kinase: A Central Role in Inflammation. Curr Top Med Chem. 2005;5:921-8.

29. Krabbe KS, Nielsen AR, Krogh-Madsen R, Plomgaard P, Rasmussen P, Erikstrup C, et al. Brain-derived neurotrophic factor (BDNF) and type 2 diabetes. Diabetologia. 2007;50:431-8.

30. Yirmiya R, Goshen I. Immune modulation of learning, memory, neural plasticity and neurogenesis. Brain Behav Immun. 2011;25:181-213.

31. Yamada T, Horisberger MA, Kawaguchi N, Moroo I, Toyoda T. Immunohistochemistry using antibodies to a-interferon and its induced protein, MxA, in Alzheimer's and Parkinson's disease brain tissues. Neuroscience Letters. 1994;181(1-2):61-4.

32. Rook GAW, Rogelio Hernandez-Pando R. The pathogenesis of tuberculosis. Annu Rev Microbiol. 1996;50(1):259-84.

33. Chan J, Xing Y, Magliozzo R, Bloom B. Killing of virulent Mycobacterium tuberculosis by reactive nitrogen intermediates produced by activated murine macrophages. J Exp Med. 1992;175(4):111122. 
34. Seah GT, Scott GM, Rook GAW. Type 2 Cytokine Gene Activation and Its Relationship to Extent of Disease in Patients with Tuberculosis. J Infect Dis. 2000;181:385-9.

35. Wangoo A, Sparer T, Brown IN, Snewin VA, Janssen R, Thole J, et al. Contribution of Th1 and Th2 Cells to Protection and Pathology in Experimental Models of Granulomatous Lung Disease. J Immunol. 2001;166:3432-9.

36. Hernandez-Pando R, Pavön L, Arriaga K, Orozco H, Madrid-Marina V, Rook G. Pathogenesis of tuberculosis in mice exposed to low and high doses of an environmental mycobacterial saprophyte before infection. Infect Immun. 1997;65:3317-27.

37. Banks W, Kastin A, Broadwell R. Passage of cytokines across the blood-brain barrier. Neuroimmunomodulation. 1995;24:241-8.

38. Katsuura G, Arimura A, Koves K, Gottschall PE. Involvement of organum vasculosum of lamina terminalis and preoptic area in interleukin $1 \beta$-induced $A C T H$ release. Am J Physiol - Endocrinol Metab. 1990;258.

39. Watkins LR, Maier SF, Goehler LE. Cytokine-to-brain communication: A review \& analysis of alternative mechanisms. Life Sci. 1995;57:1011-26.

40. Hanisch UK. Microglia as a source and target of cytokines. Glia. 2002;40:140-55.

41. Hamby ME, Sofroniew MV. Reactive astrocytes as therapeutic targets for CNS disorders. Neurotherapeutics. 2010;7(4):494-506.

42. Fabry Z, Fitzsimmons KM, Herlein JA, Moninger TO, Dobbs MB, Hart MN. Product ion of the cytokines interleukin 1 and 6 by murine brain microvessel endothelium and smooth muscle pericytes. $J$ Neuroimmunol. 1993;47:23-34.

43. Matsumura K. Signaling the brain in inflammation: the role of endothelial cells. Frontiers in Bioscience. 2004;9(1-3):2819.

44. Dantzer R. Cytokine, Sickness Behavior, and Depression. Neurol Clin. 2006;24:441-60.

45. Zandio M, Ferrín M, Cuesta MJ. Neurobiology of depression. ANALES Sis San Navarra. 2002;25(3):43-62.

46. Tank AW, Wong DL. Peripheral and central effects of circulating catecholamines. Compr Physiol. 2015;5:1-15.

47. Benarroch EE. The locus ceruleus norepinephrine system: Functional organization and potential clinical significance. Neurology. 2009;73:1699-704.

48. Feinstein DL, Heneka MT, Gavrilyuk V, Dello Russo C, Weinberg G, Galea E. Noradrenergic regulation of inflammatory gene expression in brain. Neurochem Int. 2002;41:357-65.

49. Ressler KJ, Nemeroff CB. Role of norepinephrine in the pathophysiology and treatment of mood disorders. Biol Psychiatry. 1999;46:1219-33.

50. Felger JC, Miller AH. Cytokine effects on the basal ganglia and dopamine function: The subcortical source of inflammatory malaise. Front Neuroendocrinol. 2012;33:315-27. 
51. Shuto H, Kataoka Y, Horikawa T, Fujihara N, Oishi R. Repeated interferon-a administration inhibits dopaminergic neural activity in the mouse brain. Brain Res. 1997;747:348-51.

52. De Miranda AS, Lacerda-Queiroz N, de Carvalho Vilela M, Rodrigues DH, Rachid MA, Quevedo J, et al. Anxiety-like behavior and proinflammatory cytokine levels in the brain of C57BL/ 6 mice infected with Plasmodium berghei (strain ANKA). Neurosci Lett. 2011;491:202-6.

53. de Queiroz ML, Viel TA, Papa CHG, Lescano SAZ, Chieffı PP. Alterações comportamentais em Rattus norvegicus coinfectados por Toxocara canis e Toxoplasma gondii. Rev Inst Med Trop Sao Paulo. 2013;55:51-3.

54. Corrêa FM, Chieffı PP, Lescano SAZ, dos Santos SV. Alterações comportamentais e na memória de Mus musculus coinfectado por Toxocara canis e Toxoplasma gondii. Rev Inst Med Trop Sao Paulo. 2014;56:353-6.

55. Moreau M, André C, O'Connor JC, Dumich SA, Woods JA, Kelley KW, et al. Inoculation of Bacillus Calmette-Guerin to mice induces an acute episode of sickness behavior followed by chronic depressive-like behavior. Brain Behav Immun. 2008;22:1087-95.

56. O'Connor JC, André C, Wang Y, Lawson MA, Szegedi SS, Lestage J, et al. Interferon-y and tumor necrosis factor-a mediate the upregulation of indoleamine 2,3-dioxygenase and the induction of depressive-like behavior in mice in response to bacillus calmette-guérin. J Neurosci. 2009;29:4200-9.

57. Rodriguez-Zas SL, Nixon SE, Lawson MA, Mccusker RH, Southey BR, O'Connor JC, et al. Advancing the understanding of behaviors associated with Bacille Calmette Guérin infection using multivariate analysis. Brain Behav Immun. 2015;44:176-86.

58. Kelley KW, O'Connor JC, Lawson MA, Dantzer R, Rodriguez-Zas SL, McCusker RH. Aging leads to prolonged duration of inflammation-induced depression-like behavior caused by Bacillus CalmetteGuérin. Brain Behav Immun. 2013;32:63-9.

59. Newhouse K, Hsuan SL, Chang SH, Cai B, Wang Y, Xia Z. Rotenone-induced apoptosis is mediated by p38 and JNK MAP kinases in human dopaminergic SH-SY5Y cells. Toxicol Sci. 2004;79:137-46.

60. Tamagno E, Guglielmotto M, Giliberto L, Vitali A, Borghi R, Autelli R, et al. JNK and ERK1/2 pathways have a dual opposite effect on the expression of BACE1. Neurobiol Aging. 2009;30:1563-73.

61. Cho JE, Park S, Cho SN, Lee H, Kim YS. C-Jun n-terminal kinase (JNK) and p38 mitogen-activated protein kinase (p38 MAPK) are involved in mycobacterium tuberculosis-induced expression of leukotactin-1. BMB Rep. 2012;45:583-8.

62. Bonni A, Brunet A, West AE, Datta SR, Takasu MA, Greenberg ME, et al. Cell Survival Promoted by the Ras-MAPK Signaling Pathway by Transcription- Dependent and -Independent Mechanisms. American Association for the Advancement of Science. 2016;286:1358-62.

63. Hetman M, Gosdz A. Role of extracellular signal regulated kinases 1 and 2 in neuronal survival. Eur $\mathrm{J}$ Biochem. 2004;271:2050-5.

64. Zheng Y, Fang W, Fan S, Liao W, Xiong Y, Liao S, et al. Neurotropin inhibits neuroinflammation via suppressing NF-KB and MAPKs signaling pathways in lipopolysaccharide-stimulated BV2 cells. J Pharmacol Sci. 2018;136:242-8. 
65. Wolf SA, Fisher J, Bechmann I, Steiner B, Kwidzinski E, Nitsch R. Neuroprotection by T-cells depends on their subtype and activation state. J Neuroimmunol. 2002;133:72-80.

66. Butovsky O, Talpalar AE, Ben-Yaakov K, Schwartz M. Activation of microglia by aggregated $\beta$-amyloid or lipopolysaccharide impairs MHC-II expression and renders them cytotoxic whereas IFN-y and IL-4 render them protective. Mol Cell Neurosci. 2005;29:381-93.

67. Hendrix S, Nitsch R. The role of T helper cells in neuroprotection and regeneration. J Neuroimmunol. 2007;184:100-12.

68. Meisel C, Schwab JM, Prass K, Meisel A, Dirnagl U. Central nervous system injury-induced immune deficiency syndrome. Nat Rev Neurosci. 2005;6:775-86.

69. Lehrmann E, Kiefer R, Christensen T, Toyka KV, Zimmer J, Diemer NH, et al. Microglia and macrophages are major sources of locally produced transforming growth factor- $\beta 1$ after transient middle cerebral artery occlusion in rats. Glia. 1998;24:437-48.

\section{Figures}




\section{A) Hypothalamus}

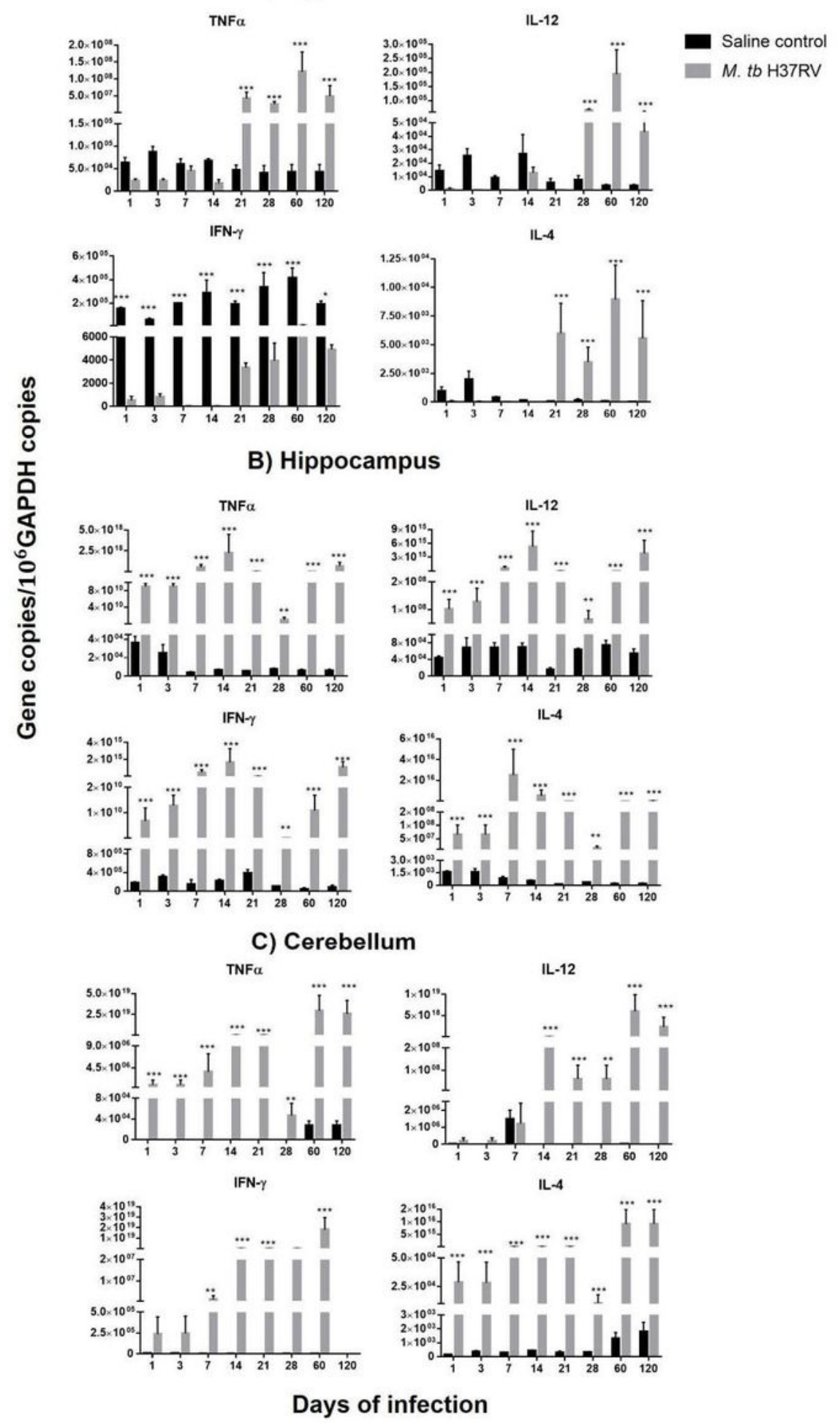

Figure 1

Cytokines expression in the hypothalamus (A), hippocampus (B) and cerebellum (C) of TB mice. Quantitative expression of mRNA for the indicated cytokines was determined by real-time PCR. Data are expressed as mean \pm SEM $(n=6) .{ }^{*} p<0.05$ and $* \star \star p<0.001$ compared to the saline control non-infected mice. 

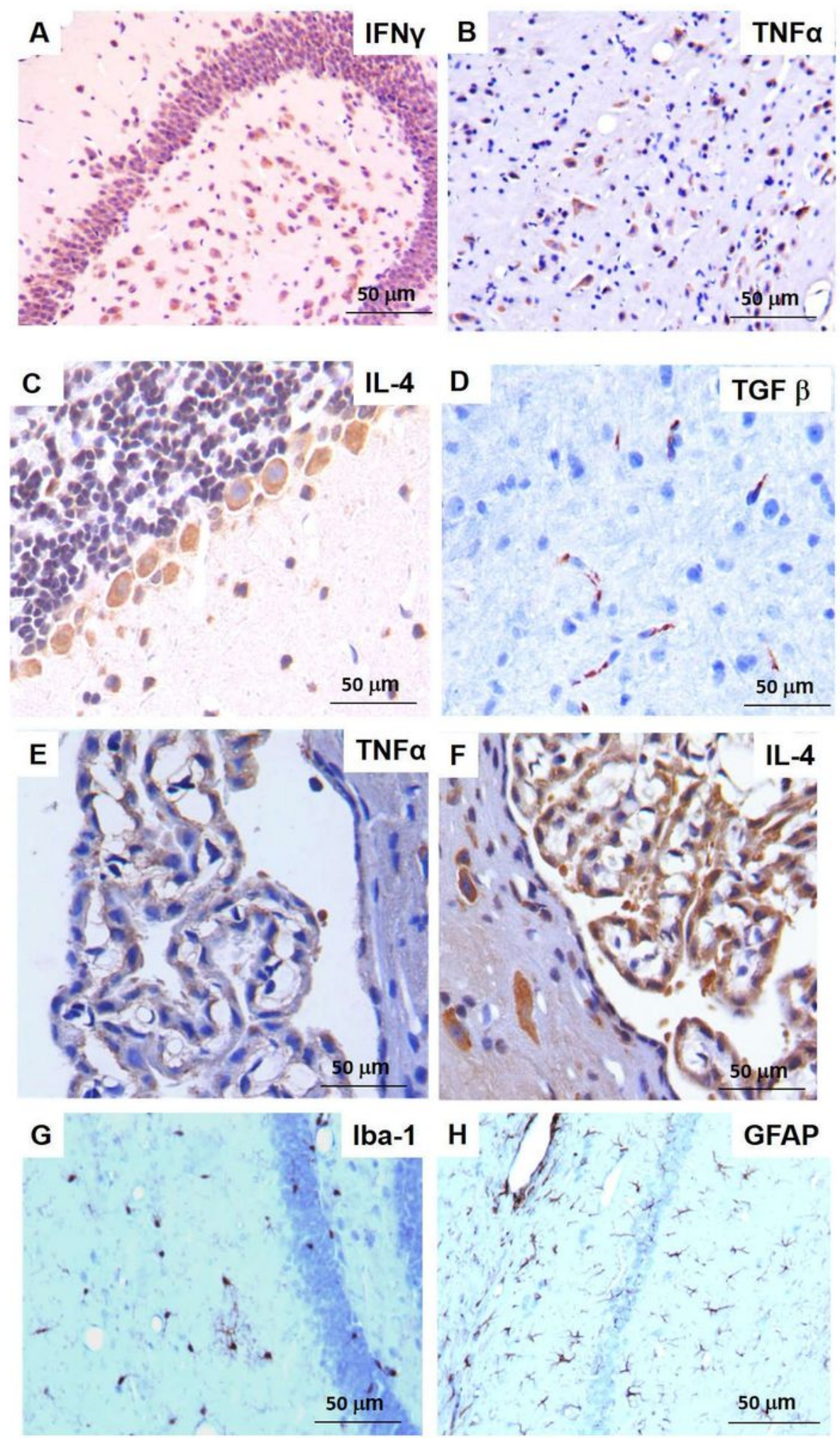

\section{Figure 2}

Detection of cytokines, lba-1 and GFAP in brain areas of TB mice after 60 days. (A) Numerous glial and neurons from hippocampus show immunostaining to IFNy. (B) Neurons and glial cells exhibit strong TNFa immunostaining in the hypothalamus. (C) Purkinje cells from cerebellum are strongly labelled for IL-4. (D) Microglia and capillary endothelial cells show selective TGF $\beta$ immunostaining. Epithelial cells from choroidal plexus show immunostaining to the pro-inflammatory cytokine TNFa (E) and anti- 
inflammatory cytokine IL-4 (F). Hippocampus show activated microglia (G) and gliosis $(H)$. Micrographs 200x magnification.
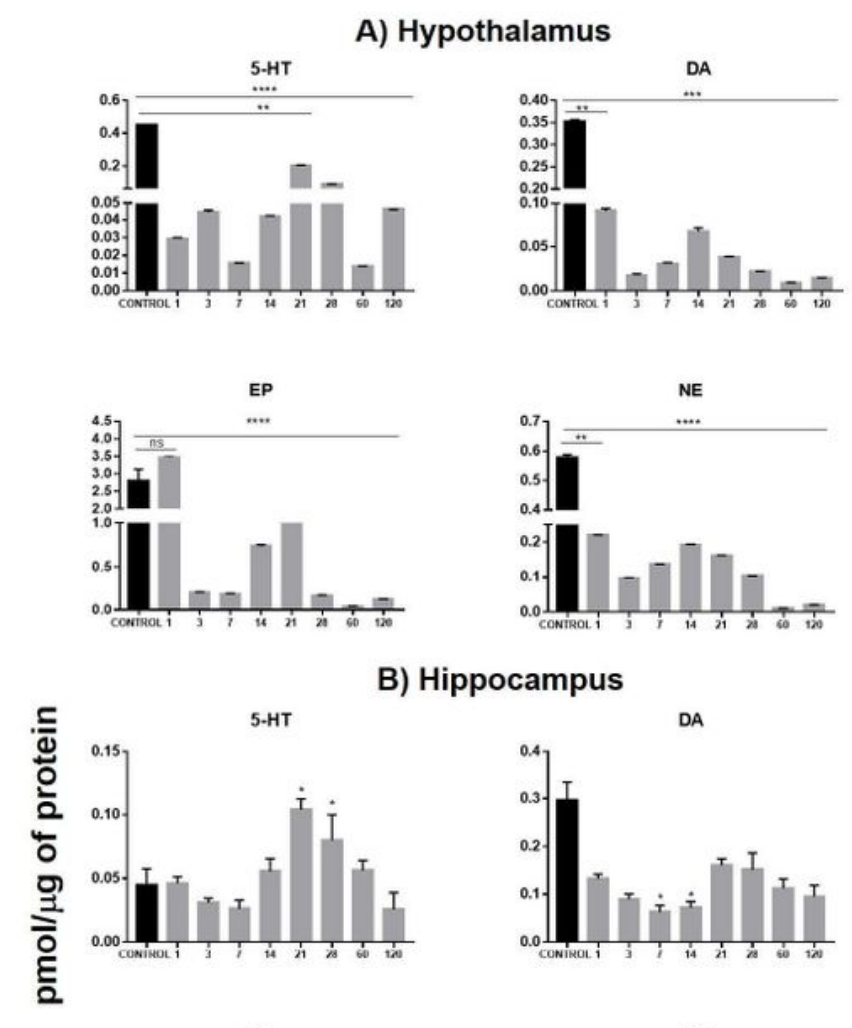

B) Hippocampus
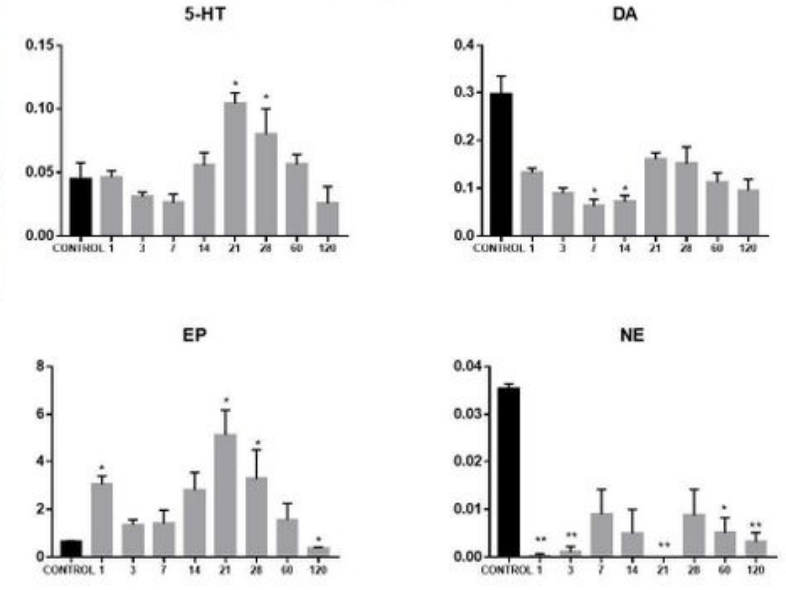

C) Cerebellum
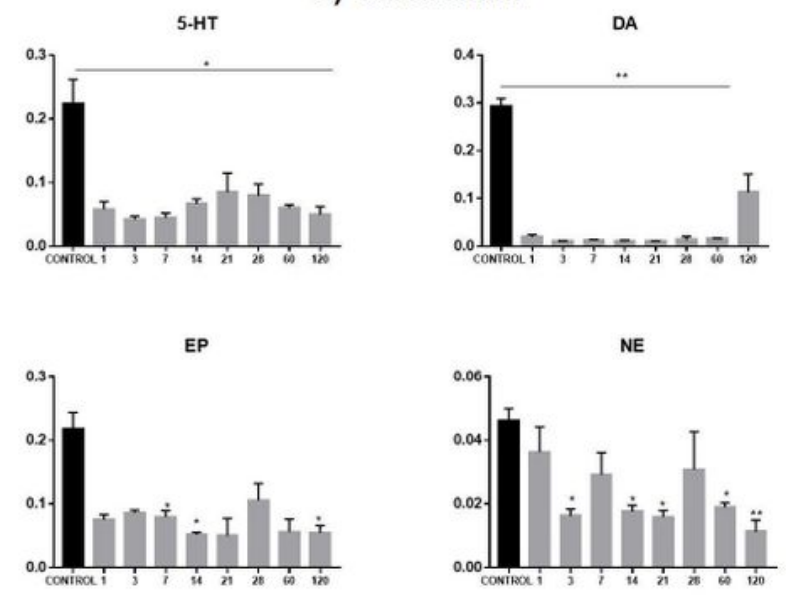

\section{Days of infection}

\section{Figure 3}

Neurotransmitters levels in the hypothalamus (A), hippocampus (B) and cerebellum (C) of TB mice. Groups of three animals in two independent experiments were euthanized in the indicated days; the hypothalamus, hippocampus and cerebellum were dissected and used to determine the 5-HT, DA, EP and 
NE. Data are expressed as mean $\pm \operatorname{SEM}(n=6)$. ${ }^{*} p<0.05, * \star p<0.005, * \star \star p<0.001$, and ${ }^{\star \star * *} p<0.0001$ compared to saline control non-infected control mice.

A) Food Intake

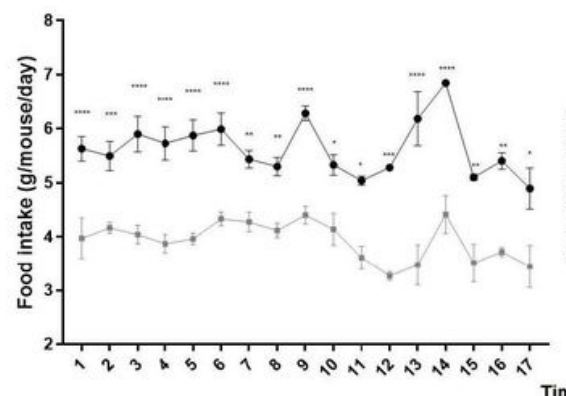

Time (weeks)
B) Loss of body weight

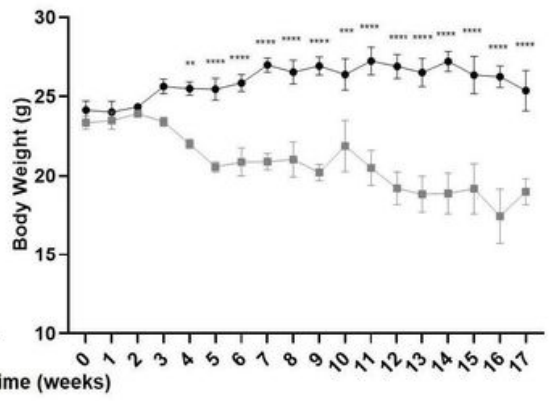

E) Anxiety like behaviour
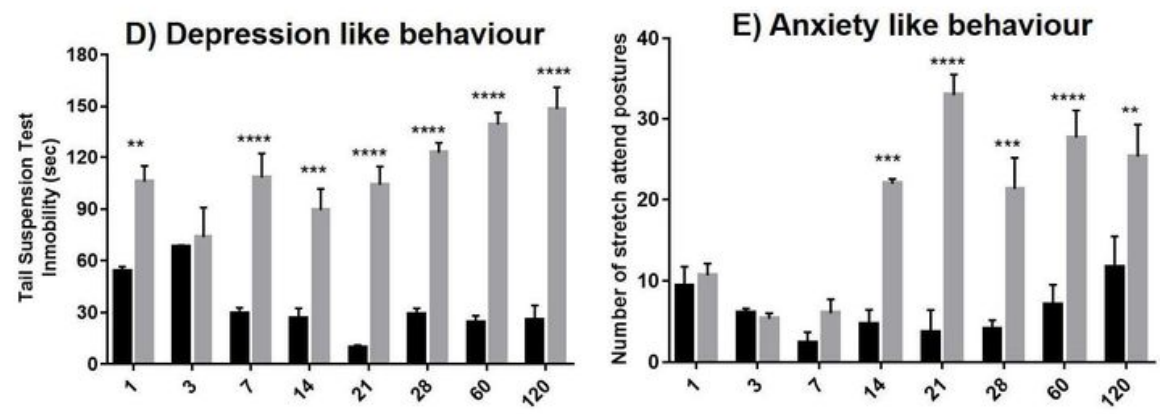

C) Locomotor Activity

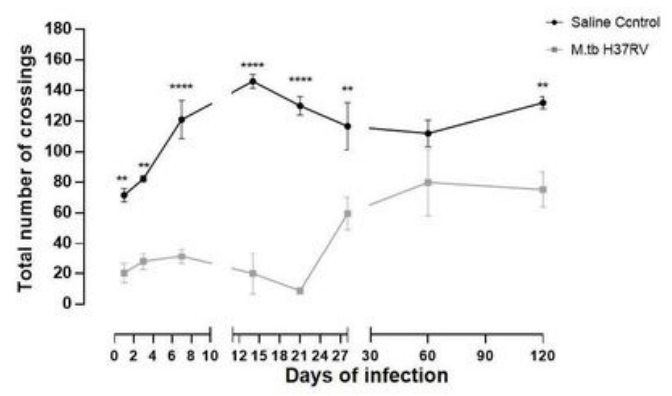

F) Neurological outcome
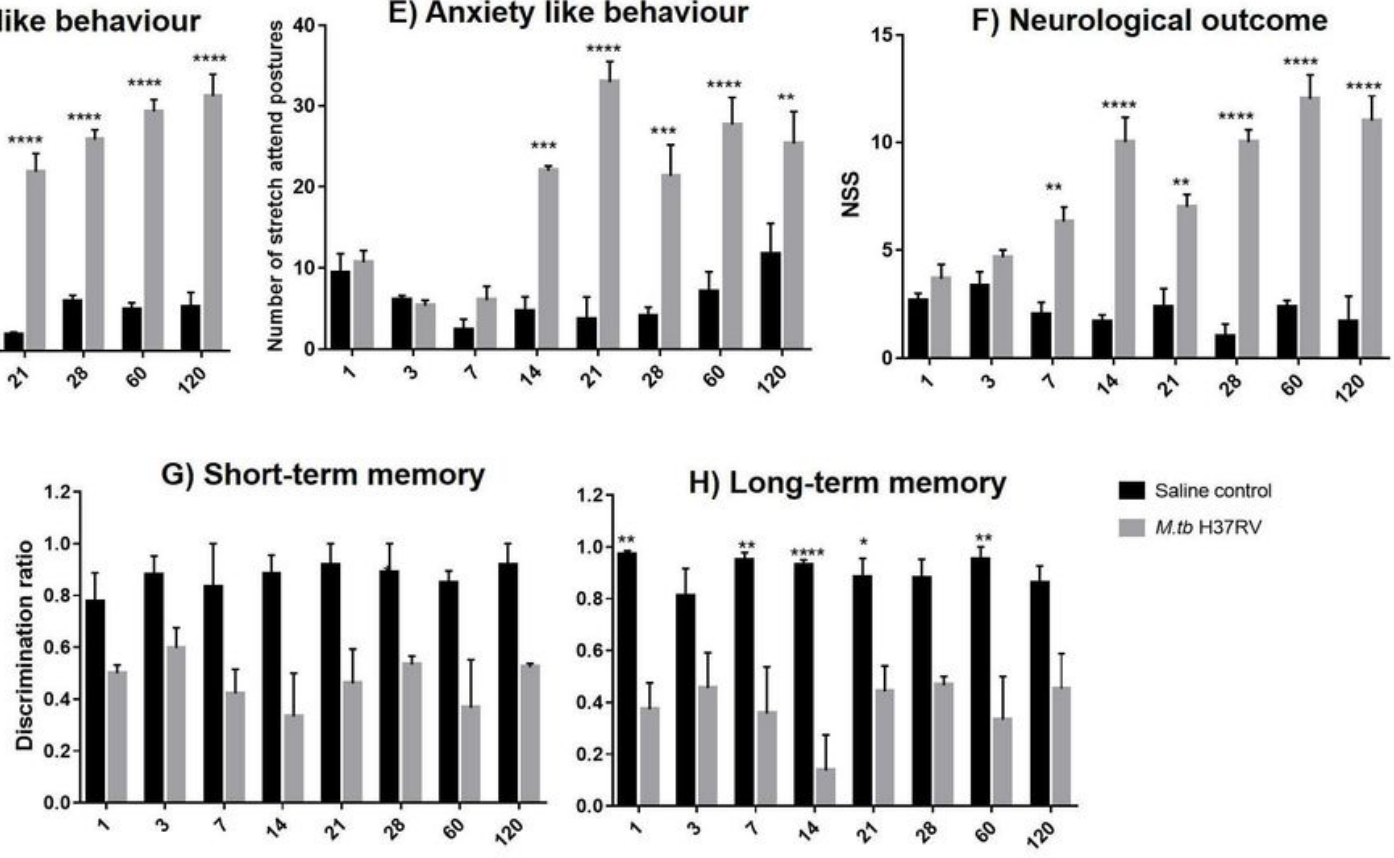

Saline contro M.tb H37RV

Days of infection

Figure 4

Behaviour abnormalities showed by TB mice. (A) Food intake, (B) Bodyweight loss and (C) Locomotor activity as characteristics of Sickness behaviour. (D) Depression-like behaviour, (E) Anxiety-like behaviour, (F) Neurological outcome, (G) Short term and (H) Long term memory. Data are expressed as mean \pm SEM $(n=6) .{ }^{*} p<0.05,{ }^{* \star} p<0.005, * \star * p<0.001$, and ${ }^{* \star * \star} p<0.0001$ compared to the saline control non-infected control mice. 

A) Hipoccampus
B) Hypophatamus
C) Cerebellum

p-38
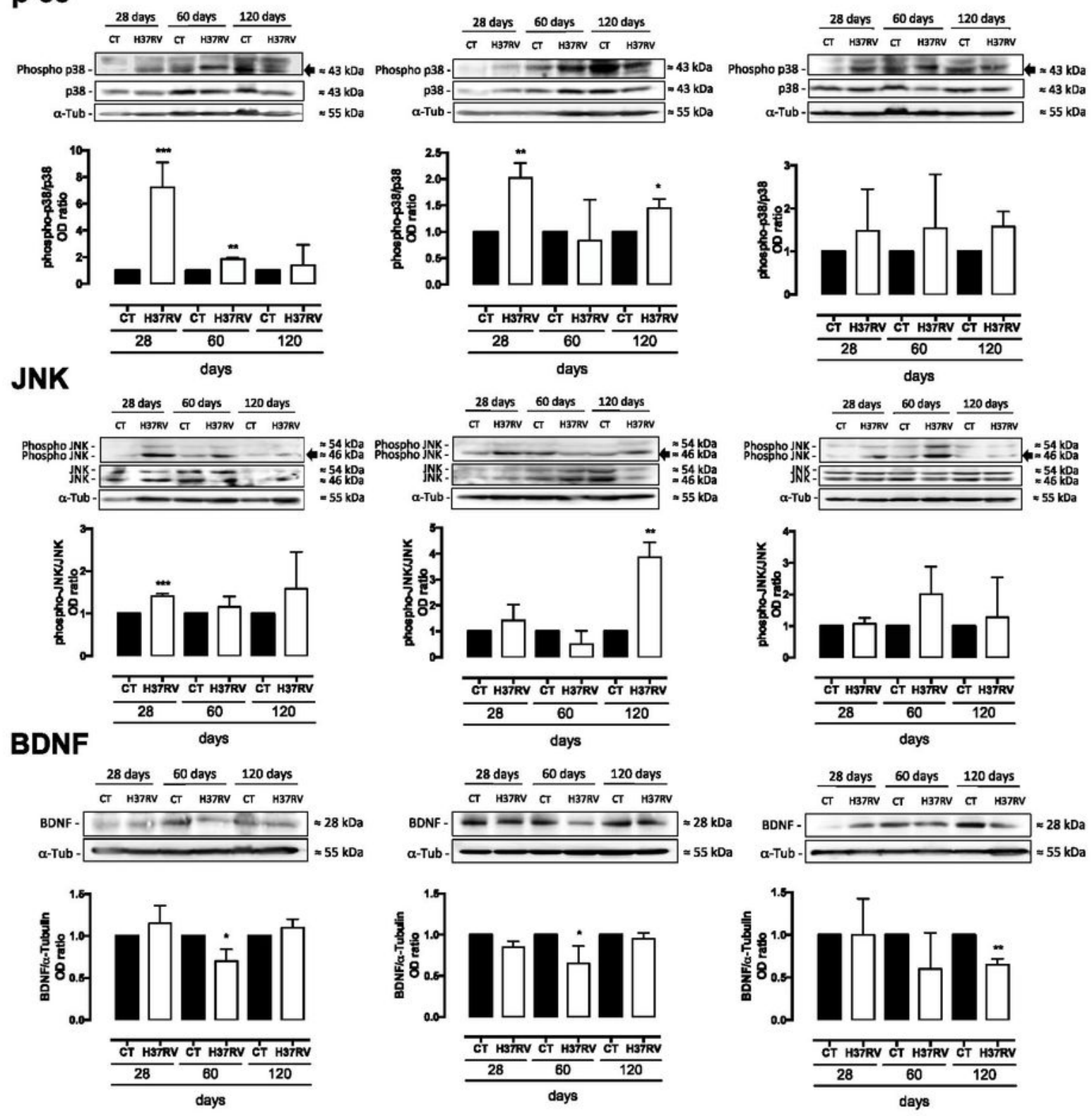

\section{Figure 5}

Expression of $\mathrm{p} 38, \mathrm{JNK}$ and BDNF in the hippocampus (A), hypothalamus (B) and cerebellum (C). The activation of $\mathrm{p} 38$ and JNK and the levels of BNDF were determined by western-blot and densitometry in TB and CT animals. Data are expressed as the mean \pm SEM. ${ }^{*} P<0.05, * * P<0.01$ and ${ }^{\star *} * P<0.001$ compared to the saline control non-infected control mice. 
A

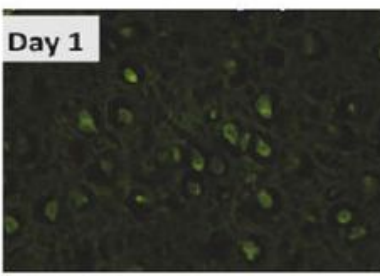

$$
\text { Day } 14
$$
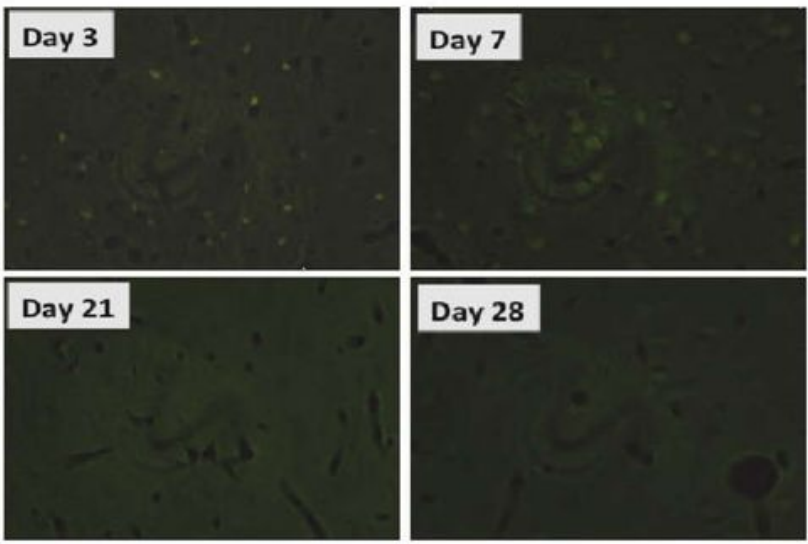

B

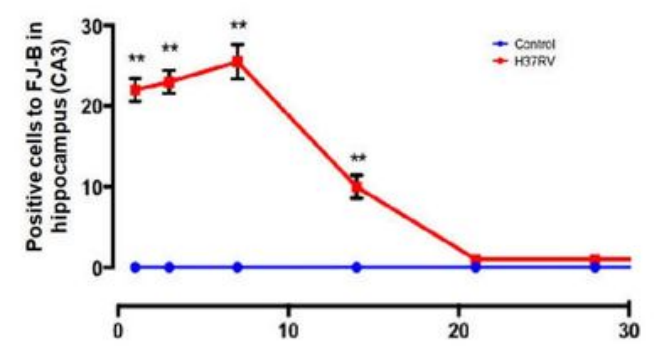

C

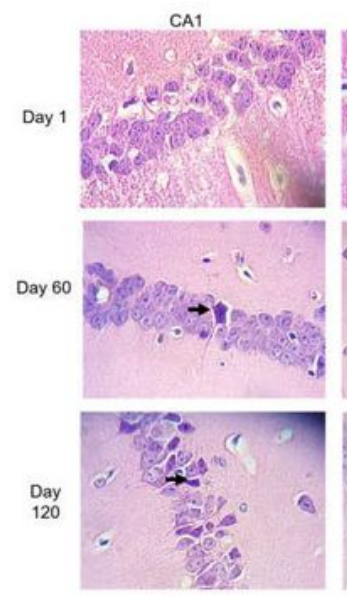

CA1

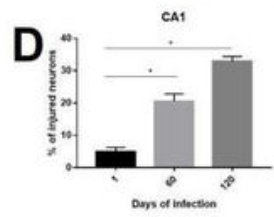

CA3

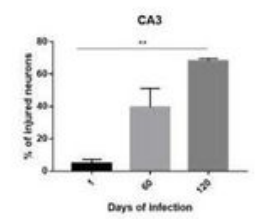

CA2

CA3

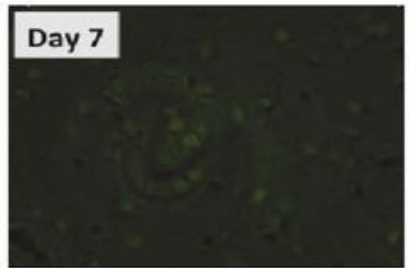

Day 28
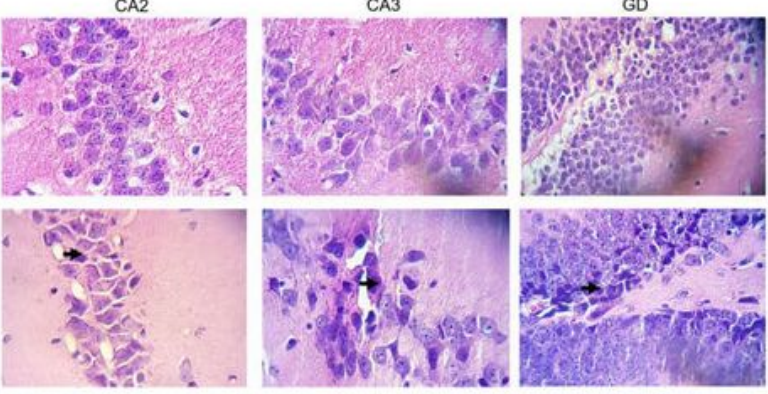

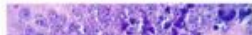
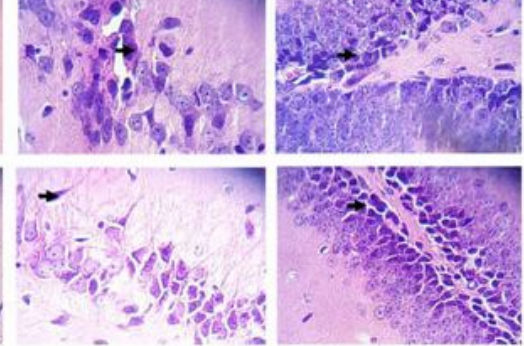

$\mathrm{CA} 2$

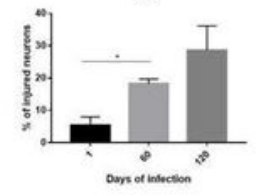

DO
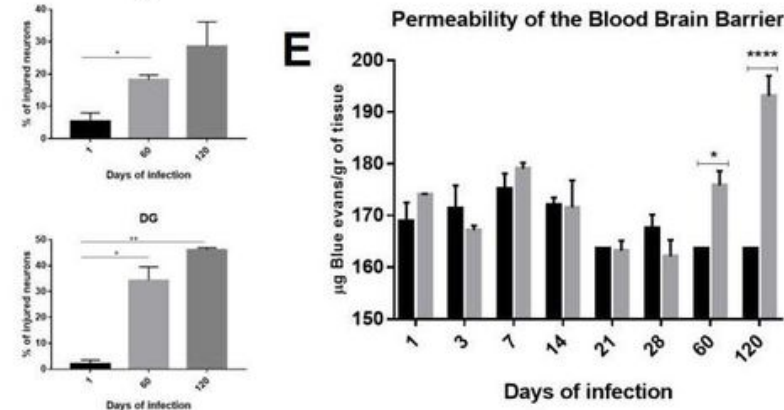

- Saline control M.tb H37RV

\section{Figure 6}

Neuronal damage, histological changes in the hippocampus and BBB dysfunction in TB mice. Neuronal damage was detected by FJ-B in BALB/c mice with pulmonary infection with $M$. tuberculosis $A$ ) representative images of the FJ-B staining in CA3 at 1, 3, 7, 14, 12, and 28 post-infection. B) Quantification of positive cells to FJ-B in the hippocampus at the same times post-infection. C) Cytological changes include neurons with acidophilic cytoplasm and fragmented (arrows) or pyknotic 
nuclei by hematoxylin/eosin staining, which were more common at day 60 post-infection. D) Percentage of injured neurons in hippocampal regions. E) BBB dysfunction in mice pulmonary infected with M. tuberculosis, as assessed by Evans blue extravasation into brain tissue. Data are expressed as mean \pm $\operatorname{SEM}(n=6) .{ }^{*} p<0.05, * * p<0.005, * \star * p<0.001$, and ${ }^{* \star * *} p<0.0001$.
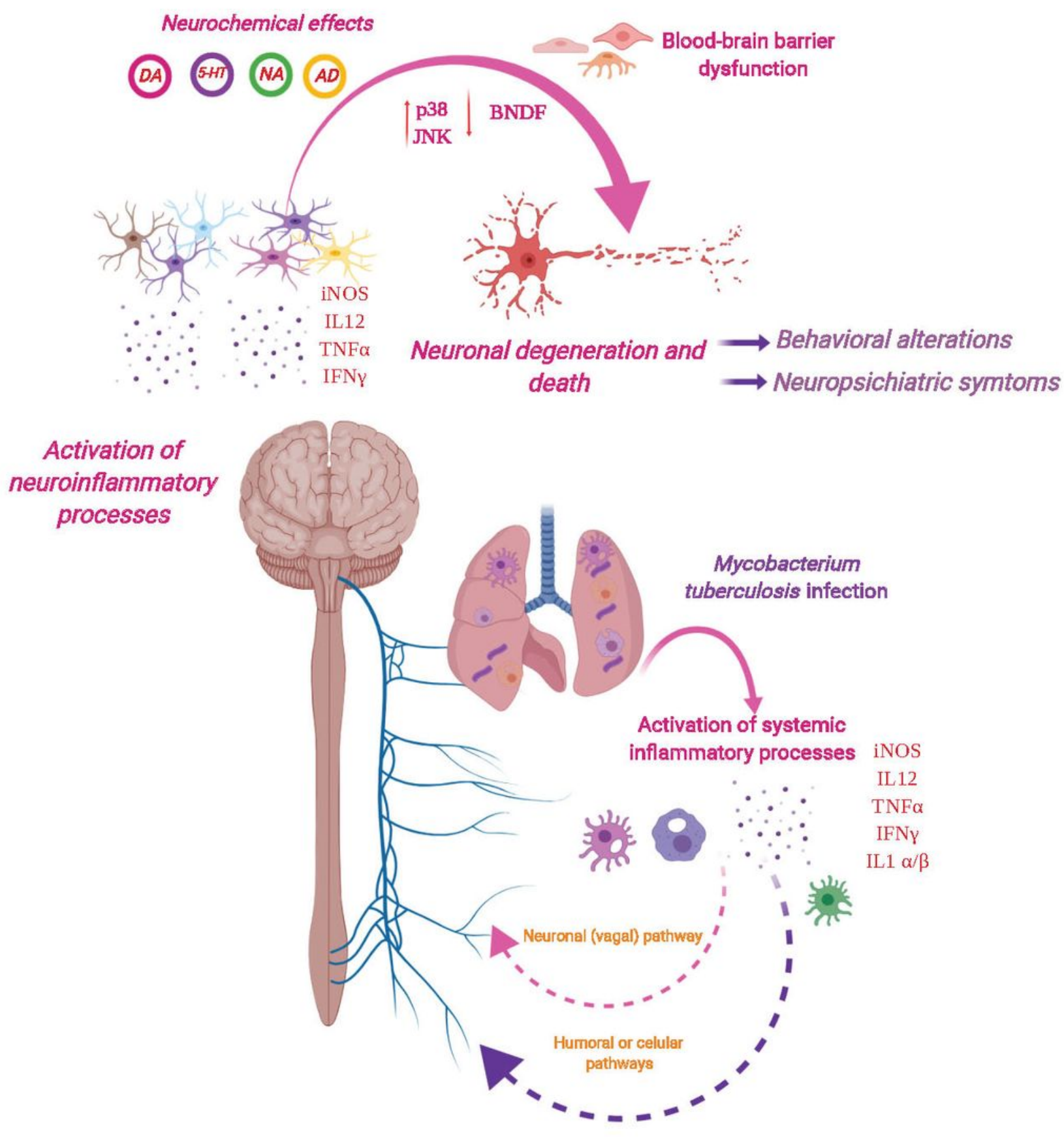

Figure 7

Mycobacterium tuberculosis induces neuroinflammation, neuronal damage and behavioural abnormalities during pulmonary infection. Activation of systemic inflammatory processes because of the 
immune response against the mycobacteria in the lungs affects the brains by neuronal and humoral pathways. Peripheral inflammation activates neuroinflammatory processes with the production of different cytokines that disturbs the central response altering the production of neurotransmitters. Even more, the central inflammatory response induces p38 and JNK activation and decreases BNDF levels. All this cause neuronal degeneration and death and increases in the permeability of the BBB that induce behavioural alterations in the infected mice (Created with BioRender).

\section{Supplementary Files}

This is a list of supplementary files associated with this preprint. Click to download.

- Additionalfile3.pdf

- Additionalfile2.pdf

- Additionalfile1.pdf 